\title{
Monotone traveling waves for delayed neural field equations
}

\author{
Jian Fang ${ }^{1}$ and Grégory Faye ${ }^{2}$ \\ ${ }^{1}$ Department of Mathematics, Harbin Institute of Technology Harbin 150001, China \\ ${ }^{2}$ CNRS, UMR 5219, Institut Mathématiques de Toulouse, 31062 Toulouse Cedex, France
}

May 31, 2016

\begin{abstract}
We study the existence of traveling wave solutions and spreading properties for single-layer delayed neural field equations. We focus on the case where the kinetic dynamics are of monostable type and characterize the invasion speeds as a function of the asymptotic decay of the connectivity kernel. More precisely, we show that for exponentially bounded kernels the minimal speed of traveling waves exists and coincides with the spreading speed, which further can be explicitly characterized under a KPP type condition. We also investigate the case of algebraically decaying kernels where we prove the non-existence of traveling wave solutions and show the level sets of the solutions eventually locate in between two exponential functions of time. The uniqueness of traveling waves modulo translation is also obtained.
\end{abstract}

\section{Introduction}

We consider the following single-layer delayed neural field equation

$$
\partial_{t} u(t, x)=-u(t, x)+\int_{\mathbb{R}} \mathcal{K}(x-y) S(u(t-\tau, y)) \mathrm{d} y, \quad(t, x) \in(0, \infty) \times \mathbb{R},
$$

where $u(t, x)$ stands for the average potential membrane of a piece of cortex at position $x$ and time $t$. Here, we consider that the neural network is distributed over the real line. In this model, the firing rate of neurons $S(u(t-\tau, x))$ is a nonlinear function of the average potential membrane which incorporates synaptic delays via the positive constant $0 \leq \tau<\infty$. The kernel $\mathcal{K}$ encodes the connectivity of the underlying network and we assume that the connections between neurons are excitatory. Neural field models were introduced at the end of the last century $[2,46]$ and have been very successful in reproducing a number of phenomena, including in particular visual hallucination patterns, binocular rivalry and working memory. We refer to the recent surveys $[5,6,12]$ for more developments on neural field models. Equations such as (1.1) can also support a rich repertoire of phenomena, such as traveling waves, spatially periodic patterns, oscillatory dynamics and localized activity [6]. Finally, note that adding delays into neural field models is a very recent development in 
the mathematical neuroscience community where most studies have focused so far on the stability and bifurcation analysis of stationary solutions of (1.1) (see [33, 39-42]).

In the present study, we would like to address two important questions. Let suppose that there exist two homogeneous stationary states $u=0$ and $u=1$ for the dynamics of (1.1). The first question is about the existence, uniqueness and qualitative properties of traveling wave solutions which connect these states monotonically. Hence, we are interested in solutions $u(t, x)=w(x-c t)=w(\xi)$, for $c \in \mathbb{R}$, where $w$ is monotone and $0 \leq w \leq 1$, which satisfy

$$
-c \frac{\mathrm{d}}{\mathrm{d} \xi} w(\xi)=-w(\xi)+\int_{\mathbb{R}} \mathcal{K}\left(\xi-\xi^{\prime}\right) S\left(w\left(\xi^{\prime}+c \tau\right)\right) \mathrm{d} \xi^{\prime},
$$

together with the limits

$$
\lim _{\xi \rightarrow-\infty} w(\xi)=1 \text { and } \lim _{\xi \rightarrow+\infty} w(\xi)=0 .
$$

The second question is related to the spreading properties of system (1.1) and can easily be formulated as follows: what happens to an initially compactly supported initial condition, bounded by the two homogeneous stationary states $u=0$ and $u=1$, under the time evolution of (1.1)? Theses two questions are crucial from a modeling point of view. Indeed, in the past few years, electrode recordings and imaging studies have revealed that the primary visual cortex can support a variety of cortical waves including standing waves [4, 34], traveling pulses [4, 29, 48] and spiral waves $[24,25,47]$. These traveling waves are not only elicited by localized visual stimuli across the visual cortex but they are also present during spontaneous activity [25, 34]. It is thus important to understand the underlying mechanisms which allow the propagation of coherent structures such as traveling waves. In this study, we will focus ourselves to the case of spontaneous activity in the sense that our model equation (1.1) does not include any external input.

The mathematical study of traveling waves for neural field equations goes back to the pioneering work of Ermentrout and McLeod [15] in the absence of delay and when the system is of bistable type. That is, there exists $0<a<1$ such that $S(a)=a$ and there is no other zero of $-u+S(u)=0$ in $(0,1)$, with $S^{\prime}(0)<1$ and $S^{\prime}(1)<1$. In that case, it was shown in [15] that there exists a unique monotone traveling wave (modulo translation), solution of (1.2) and (1.3), with a unique associated wave speed $c_{*} \in \mathbb{R}$. More recent works of the second author deal with the existence of traveling pulses, i.e. non-monotone traveling waves connecting the same homogeneous stationary state, for neural field models with a recovery variable, such as linear adaptation [20] or synaptic depression [21]. In the present study, we consider only systems of monostable type (see Hypothesis (H2) below for a precise statement). Formally, this means that $u=0$ and $u=1$ are the only homogeneous stationary states and $-u+S(u)>0$ in $(0,1)$. To the best of our knowledge, there are no rigorous results regarding the existence of monotone traveling waves for neural field equations of monostable type, except maybe the two formal studies $[7,13]$.

It is interesting to notice that the neural field equation (1.1) have certain similarities with timedelayed nonlocal reaction-difffusion equations arising from age-structured population dynamics 
which takes the form

$$
\partial_{t} v(t, x)=D \partial_{x x}^{2} v(t, x)-d v(t, x)+\mu \int_{\mathbb{R}} \mathcal{G}_{\alpha}(x-y) b(v(t-\tau, y)) \mathrm{d} y, \quad(t, x) \in(0, \infty) \times \mathbb{R} .
$$

The above equation (1.4) describes a diffusive single-species population with two stages of age, where $v(t, x)$ denotes the total mature population at time $t$ and position $x, D \geq 0$ denotes the diffusion coefficient, $d>0$ denotes the death rate and $\mu$ denotes the survival rate from newborn to being adult. The birth function $b(v)$ is a nonlinear function of the total mature population and $\mathcal{G}_{\alpha}$ is taken to be the heat kernel

$$
\mathcal{G}_{\alpha}(x):=\frac{1}{4 \pi \alpha} e^{-x^{2} / 4 \alpha} .
$$

We refer to [36] for the original derivation of (1.4) and more biological interpretations (see [49] for the case where $D=0$ and [30] for the bistable case). One may observe that the interaction kernel $\mathcal{G}_{\alpha}$ goes to $\delta(x)$ as $\alpha \rightarrow 0$. In that limit, neglecting the delay, we recover the standard local reaction-diffusion equation $[22,26]$

$$
\partial_{t} v(t, x)=D \partial_{x x}^{2} v(t, x)+g(v(t, x)), \quad(t, x) \in(0, \infty) \times \mathbb{R},
$$

for which we have the following well-known results from Aronson \& Weinberger [3] and Weinberger [43].

Theorem 1 ([3, 43]). Assume that $g(0)=g(1)=0$ with $g(v)>0, v \in(0,1)$ and $g^{\prime}(0)>0$. Let $v$ be a solution of $(1.5)$ with $u(0, \cdot) \neq 0$ compactly supported in $\mathbb{R}$ and satisfying $0 \leq u(0, \cdot) \leq 1$. Then, there exists $c_{*} \in(0,+\infty)$ such that

(i) if $c>c_{*}$, then $u(t, x) \rightarrow 0$ uniformly in $\{[x \mid \geq c t\}$ as $t \rightarrow \infty$;

(ii) if $c<c_{*}$, then $u(t, x) \rightarrow 1$ uniformly in $\{[x \mid \leq c t\}$ as $t \rightarrow \infty$.

Here $c_{*}$ is the minimal speed of traveling wave solutions having the form $v(t, x)=\varphi(x-c t)$ and satisfying

$$
D \varphi^{\prime \prime}+c \varphi^{\prime}+g(\varphi)=0 \text { in } \mathbb{R}, \quad \varphi(-\infty)=1, \quad \varphi(+\infty)=0 .
$$

If, in addition, $g(v) \leq g^{\prime}(0) v, v \in[0,1]$, then $c_{*}=2 \sqrt{D g^{\prime}(0)}$.

We expect in this paper to find conditions, especially on the connectivity kernel, such that a similar result to Theorem 1 holds or does not hold for the neural field equation (1.1). In a recent work [18], an age-structured population model of Asian clams has been investigated by the first author and his collaborators in which the coefficient diffusion in (1.4) is taken to be $D=0$ and an additional population is introduced in order to take into account the sperm population. The main results of [18] suggest that (1.1) with heat connectivity kernel should have the same spreading property as stated in Theorem 1. Both (1.1) and (1.4) could be transferred into the general integral equation investigated in [16], where the existence and uniqueness of traveling waves as well as the spreading speed were obtained subjected to a KPP type condition and exponentially 
bounded kernels. However, the spreading properties of (1.1) remains unclear for general kernels and monostable nonlinearities. Throughout the paper, we will assume that the kernel $\mathcal{K}$ and the nonlinearity $S$ satisfy the following hypotheses.

Hypothesis (H1) The function $\mathcal{K}$ defined on $\mathbb{R}$ is such that:

(i) $\mathcal{K}$ is uniformly bounded on $\mathbb{R}$;

(ii) $\mathcal{K} \geq 0$;

(iii) $\int_{\mathbb{R}} \mathcal{K}(x) \mathrm{d} x=1$.

The first condition is a natural biological assumption on the connectivity kernel, and the second assumption expresses the excitatory nature of the considered neural network. The last assumption is a normalization condition.

\section{Hypothesis (H2)}

(i) $S$ is continuously differentiable with $0<S^{\prime} \leq s_{m}$;

(ii) $f(u):=-u+S(u)$ has precisely two zeros at $u=0$ and $u=1$, with $f(u)>0$ for all $u \in(0,1)$;

(iii) $S^{\prime}(0)>1$.

The assumption (i), that $S$ is increasing, is natural for a firing rate function. The second condition together with the condition (iii) for $\mathcal{K}$ ensures that $u=0$ and $u=1$ are stationary solutions of equation (1.1). The last condition corresponds to the setting in which the states $u=0$ is unstable.

We are now in position to state the main results of the paper. The first result is about the large time behavior of solutions to the Cauchy problem

$$
\left\{\begin{aligned}
\partial_{t} u(t, x) & =-u(t, x)+\int_{\mathbb{R}} \mathcal{K}(x-y) S(u(t-\tau, y)) \mathrm{d} y & & \text { if }(t, x) \in(0, \infty) \times \mathbb{R}, \\
u(t, x) & =\phi(t, x) & & \text { if }(t, x) \in[-\tau, 0] \times \mathbb{R} .
\end{aligned}\right.
$$

Theorem 2 (Spreading speeds). The Cauchy problem (1.7) admits leftward and rightward spreading speeds, denoted by $c_{-}^{*}$ and $c_{+}^{*}$ respectively, satisfying $c_{-}^{*}+c_{+}^{*}>0$ and $c_{ \pm}^{*} \in(-\infty,+\infty]$, in the following sense:

(i) if $c>c_{+}^{*}$ and $c^{\prime}>c_{-} *$ and the initial condition $\phi$ has compact support with $0 \leq \phi \leq 1$ and $\phi \not \equiv 1$, then the solution $u$ of (1.7) has the property

$$
\lim _{t \rightarrow \infty, x \geq c t \text { or } x \leq-c^{\prime} t} u(t, x)=0 ;
$$

(ii) if $c<c_{+}^{*}$ and $c^{\prime}<c_{-}^{*}$ with $c+c^{\prime}>0$ and the initial condition $\phi$ has compact support with $0 \leq \phi \leq 1$ and $\phi \not \equiv 0$, then the solution $u$ of (1.7) has the property

$$
\lim _{t \rightarrow \infty, c^{\prime} t \leq x \leq c t} u(t, x)=1 \text {. }
$$


Remark 1.1. We readily remark that $c_{-}^{*}=c_{+}^{*}:=c^{*}$ when the connectivity kernel $\mathcal{K}$ is symmetric and both of them could be infinite in specific evolutionary models, which will be discussed later.

The second result is about the existence and uniqueness of monotone traveling wave solutions of (1.1). Recall that a leftward traveling wave with speed $c$ of (1.1) is a special solution having the form $u(t, x)=w(x+c t)$ and a rightward traveling wave with speed $c$ is defined using $u(t, x)=w(x-c t)$.

Theorem 3 (Traveling waves). Let $c_{ \pm}^{*}$ be the spreading speeds of (1.7) defined in Theorem 2. Then $c_{-}^{*}$ is the minimal wave speed of the leftward nondecreasing traveling wave connecting $u=0$ to $u=1$, and $c_{+}^{*}$ is the minimal wave speed of the rightward nonincreasing traveling wave connecting $u=1$ to $u=0$.

The proofs of Theorems 2 and 3 will rely on the application of the abstract monotone dynamical systems theory developed recently in $[17,27,28]$. It is important to note that, to the best of our knowledge, it is the first time that such results on spreading properties and traveling waves solutions are presented for neural field equations with synaptic delays and monostable kinetics. The leftward or rightward spreading speeds obtained in Theorem 2 might be infinite. This actually depends on the asymptotic decaying properties of the connectivity kernel $\mathcal{K}$ (see Theorem 6 ). We first summarize in the following theorem the results on the characterization of the minimal wave speed for exponentially bounded kernels. We refer to Section 3 for more precise statements.

Theorem 4 (Characterization of the minimal wave speed). Let suppose that the initial condition $\phi$ has compact support with $0 \leq \phi \leq 1$ and $\phi \not \equiv 0$ and that $\mathcal{K}$ is symmetric. If the kernel $\mathcal{K}$ is exponentially bounded and the nonlinearity $S$ satisfies a KPP condition, then the minimal wave speed is bounded and can be explicitly characterized through the principal eigenvalue of the linearized equation around the unstable steady state $u=0$. Furthermore, as a function of the delay, the minimal wave speed $\tau \rightarrow c^{*}(\tau)$ is a monotone continuously decreasing function which converges to zero as $\tau \rightarrow+\infty$ (see Lemma 3.1 and Figure 1 for an illustration).

We now provide another result regarding the uniqueness of monotone traveling wave solutions given in Theorem 3 in the special case of symmetric exponentially bounded kernels $\mathcal{K}$ and nonlinearity $S$ which satisfies a KPP-like condition.

Theorem 5 (Uniqueness of traveling waves). Let suppose that $\mathcal{K}$ is a symmetric exponentially bounded kernel and that $S \in \mathcal{C}^{2}$ in a neighborhood of $u=0$. Then the monotone traveling wave solutions of (1.2) and (1.3) are unique up to translation. Furthermore if $S$ satisfies the KPP condition (3.2), each monotone traveling wave solution $u$ of (1.2) and (1.3) satisfies

$$
u(\xi) \underset{\xi \rightarrow+\infty}{=} \mathcal{O}\left(\xi e^{-\mu^{*} \xi}\right) \quad \text { for } c=c^{*}, \quad \text { and } \quad u(\xi) \underset{\xi \rightarrow+\infty}{=} \mathcal{O}\left(e^{-\mu_{1}(c) \xi}\right) \quad \text { for } c>c^{*},
$$

for some $0<\mu_{1}(c)$ and $0<\mu^{*}$.

Finally, our last results deal with kernels that are symmetric but not exponentially bounded. Our main theorem is the following one. 
Theorem 6 (Exponentially unbounded kernels). Let suppose that the initial condition $\phi$ has compact support with $0 \leq \phi \leq 1$ and $\phi \not \equiv 0$ and that $\mathcal{K}$ is symmetric.

- If the kernel $\mathcal{K}$ is exponentially unbounded, then the level sets of the solution u to (1.7) propagate with an infinite asymptotic speed.

- In the specific case of algebraically decaying kernels, the position of any level sets moves exponentially fast as time goes to infinity (see Propositions 5.2 and 5.3).

Roughly speaking, the second point of Theorem 6 can be stated as follows. Suppose that $\mathcal{K}(x)$ is defined as

$$
\mathcal{K}(x)=\frac{C_{\alpha}}{1+|x|^{2 \alpha}}, \quad \alpha>1 / 2,
$$

and $C_{\alpha}>0$ is a normalizing constant. Then, for any $\tau \geq 0$ and any $\kappa \in(0,1)$ there exists positive constants $\rho^{*}(\tau) \leq \bar{\rho}(\tau)$, characterized in Section 5 , such that

$$
\frac{\rho^{*}(\tau)}{2 \alpha} \leq \liminf _{t \rightarrow \infty} \frac{\log (\min \{x: u(t, x)=\kappa\})}{t} \leq \limsup _{t \rightarrow \infty} \frac{\log (\max \{x: u(t, x)=\kappa\})}{t} \leq \frac{\bar{\rho}(\tau)}{2 \alpha} .
$$

While our approach is built on the one developed in the work of Garnier [23], it differs greatly on a technical level. Indeed, our equation presents two main difficulties: the nonlinear part of the equation is nonlocal and we have to deal with time delays. Nonetheless, we are able to take the advantage of the strategy developed by Cabré and Roquejoffre [9] in the context of Fisher-KPP equations with fractional diffusion to establish the lower bound in (1.8). A key ingredient is a subtle estimate for the solutions of some linear nonlocal Cauchy problems with delays, which is independently of interest, see Section 5 .

Theorems 4 and 6 have several important implications from a modeling point of view. First of all, we clearly see that the asymptotic decaying properties of the connectivity kernel $\mathcal{K}$ are crucial and that one needs strong enough decay (exponential) in order to have finite spreading speeds $[11,23,37,38,43,44]$. Of course, from a neurobiological point of view [25, 34], infinite asymptotic speed does not seem plausible and have, so far, never been recorded in the literature. This result should then be interpreted as follows. In order to have a representative model of neuronal excitatory connections within cortical areas, one should use exponentially bounded kernels. Finally, we recover the fact that constant synaptic delays slow down the spreading speed of the system for exponentially bounded kernels in the case of monostable type of nonlinearity. This was already established in previous studies when the nonlinearity $S$ was idealized with a Heaviside step function, see [5, 6] and references therein.

Outline. The outline of the paper is as follows. After introducing the general abstract formalism on monotone semiflows, we prove Theorems 2 and 3 in Section 2. Section 3 is devoted to the characterization of the minimal wave speed for exponentially bounded kernels. Then, we prove Theorem 5 in Section 4. Finally, in Section 5 we prove acceleration phenomena for exponentially unbounded kernels and thoroughly study the specific case of a class of algebraically decaying kernels. We conclude with a discussion. 


\section{Spreading speeds and traveling waves}

\section{$2.1 \quad$ Notation}

Let $\Omega:=[-\tau, 0]$, where $\tau>0$ is the time delay in the model. Let $\mathcal{X}=C(\Omega, \mathbb{R})$ the set of continuous fonctions from $\Omega$ to $\mathbb{R}$. We endow $\mathcal{X}$ with the maximum norm $\|\cdot\|$ and the partial ordering induced by the positive cone $\mathcal{X}_{+}:=C\left(\Omega, \mathbb{R}_{+}\right)$. Then $\left(\mathcal{X}, \mathcal{X}_{+},\|\cdot\|\right)$ is a Banach lattice. For $\varphi_{1}, \varphi_{2} \in \mathcal{X}$, we write $\varphi_{1} \geq \varphi_{2}$ if $\varphi_{1}-\varphi_{2} \in \mathcal{X}_{+}, \varphi_{1} \gg \varphi_{2}$ if $\varphi_{1}-\varphi_{2} \in \operatorname{Int} \mathcal{X}_{+}$, and $\varphi_{1}>\varphi_{2}$ if $\varphi_{1} \geq \varphi_{2}$ but $\varphi_{1} \neq \varphi_{2}$. We use $\mathcal{C}$ to denote all continuous and bounded functions from $\mathbb{R}$ to $\mathcal{X}$. Let $\mathcal{M}$ be the space consisting of all monotone functions from $\mathbb{R}$ to $\mathcal{X}$. We endow both $\mathcal{C}$ and $\mathcal{M}$ with the compact open topology, which can be induced by the following metric

$$
d(u, v):=\sum_{k=1}^{\infty} \frac{\max _{|x| \leq k}\|u(x)-v(x)\|}{2^{k}}, \quad u, v \in \mathcal{C} .
$$

Therefore, a sequence $u_{n}$ is said to be convergent to $u$ in $\mathcal{C}$ provided that $u_{n}(x) \rightarrow u(x)$ in $\mathcal{X}$ uniformly locally in $x \in \mathbb{R}$ (that is, uniformly for $x$ in any compact subset of $\mathbb{R}$ ). For $u_{1}, u_{2} \in \mathcal{C}$, we write $u_{1} \geq u_{2}$ if $u_{1}(x) \geq u_{2}(x)$ for all $x \in \mathbb{R}$. We also define $\mathcal{X}_{b}:=\{u \in \mathcal{X} \mid 0 \leq u \leq 1\}$ together with

$$
\mathcal{C}_{b}:=\left\{u \in \mathcal{C} \mid u(x) \in \mathcal{X}_{b}, \forall x\right\}, \quad \mathcal{M}_{b}:=\left\{u \in \mathcal{M} \mid u(x) \in \mathcal{X}_{b}, \forall x\right\}
$$

Finally, a subset $U$ of $\mathcal{C}$ is bounded if $\sup _{u \in U} d(u, 0)$ is finite. For $u \in \mathcal{C}$ and closed interval $I \subset \mathbb{R}$, define the function $u_{I} \in C(I, \mathcal{X})$ by $u_{I}(x)=u(x)$ for all $x \in I$. Given a bounded set $U \subset \mathcal{C}$, we use $U_{I}$ to denote the the set $\left\{u_{I}: u \in U\right\}$. We use the Kuratowski measure to define the noncompactness of $U_{I}$ which is naturally endowed with the uniform topology. The measure is defined as follows.

$$
\alpha\left(U_{I}\right):=\inf \left\{r>0: U_{I} \text { has a finite open cover of diameter less than } r\right\} .
$$

The set $U_{I}$ is precompact if and only if $\alpha\left(U_{I}\right)=0$.

\subsection{Well posedness and comparison principle}

Lemma 2.1. For an initial condition $\phi \in \mathcal{C}_{b}$, equation (1.1) admits a unique solution $(t, x) \mapsto$ $u(t, x ; \phi)$ which exists globally in time $t \geq-\tau$, that is $t \mapsto u(t, \cdot ; \phi) \in C([-\tau ;+\infty), C(\mathbb{R}, \mathbb{R})) \cup$ $C^{1}([0 ;+\infty), C(\mathbb{R}, \mathbb{R}))$, such that $u_{0}=\phi$ and $u_{t} \in \mathcal{C}_{b}$ for $t \geq 0$, where $u_{t}$ is defined by

$$
u_{t}(\theta, x ; \phi)=u(t+\theta, x ; \phi) .
$$

Proof. The proof of existence and uniqueness of global in time solutions for (1.1) given an initial condition in $\mathcal{C}$ is well known, see [31, Corollaries 4 and 5]. Here, we only prove that given $\phi \in \mathcal{C}_{b}$ 
then the solution $u_{t} \in \mathcal{C}_{b}$ for $t \geq 0$. For this, we use an iterative argument and first solve equation (1.1) for $t \in(0, \tau]$ as follows

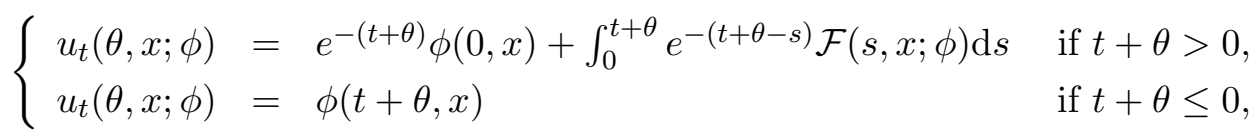

where

$$
\mathcal{F}(s, x ; \phi):=\int_{\mathbb{R}} \mathcal{K}(x-y) S(\phi(s-\tau, y)) \mathrm{d} y .
$$

Since, $\phi \in \mathcal{C}_{b}$ and $S(u)$ is nondecreasing, we have $0 \leq \mathcal{F}(s, x ; \phi) \leq 1$ for $s \in[0, \tau]$ and $\forall x \in \mathbb{R}$. As a consequence, $u_{t} \in \mathcal{C}_{b}$ for $t \in(0, \tau]$. For $t \in(\tau ; 2 \tau]$, we use the same argument shown above. By induction, equation (1.1) admits a unique solution which exists globally in time and such that $u_{t} \in \mathcal{C}_{b}$ for $t \geq 0$.

Using similar arguments, we can also obtain the well posedness on $\mathcal{M}_{b}$.

Lemma 2.2. For an initial condition $\phi \in \mathcal{M}_{b}$, equation (1.1) admits a unique solution $(t, x) \mapsto$ $u(t, x ; \phi)$ which exists globally in time $t \geq-\tau$ such that $u_{0}=\phi$ and $u_{t} \in \mathcal{M}_{b}$ for $t \geq 0$, where $u_{t}$ is defined in (2.3).

Finally, we prove a comparison principle for upper and lower solutions of (1.1). We thus introduce the following definition.

Definition 2.3. A function $\bar{u}:[-\tau, \infty) \times \mathbb{R} \rightarrow \mathbb{R}$ is said to be an upper solution of (1.1) if for $t \geq 0, u_{t} \in \mathcal{C}_{b}$ and

$$
\bar{u}(t, x) \geq e^{-t} \bar{u}(0, x)+\int_{0}^{t} e^{-(t-s)} \mathcal{F}(s, x, \bar{u}) \mathrm{d} s .
$$

A lower solution is defined by reversing the inequality in (2.4).

Using similar techniques as in the above lemma, we obtain the following comparison principle.

Lemma 2.4. If $\bar{u}$ and $\underline{u}$ are a pair of upper and lower solutions of (1.1) with $\bar{u}_{0} \geq \underline{u}_{0}$, then $\bar{u}_{t} \geq \underline{u}_{t}$ for $t \geq 0$.

\subsection{Abstract results on semiflows}

We start this section by recalling the definition of time-continuous semiflow.

Definition 2.5. A family of mapping $\left\{Q_{t}\right\}_{t \geq 0}$ is said to be a semiflow on $\mathcal{C}_{b}$ if the following properties hold:

(i) $Q_{0}=I$, where $I$ is the identity mapping;

(ii) $Q_{t} Q_{s}=Q_{t+s}$ for $t, s \geq 0$;

(iii) $Q_{t}[u]$ is continuous in $(t, u) \in[0, \infty) \times \mathcal{C}_{b}$. 
We define the translation operator $\mathcal{T}$ by $\mathcal{T}_{y}[u](x)=u(x-y)$ for $(x, y) \in \mathbb{R}^{2}$ Let $Q: \mathcal{C}_{b} \longrightarrow \mathcal{C}_{b}$ be a given map. The following assumptions on map $Q$ will be referred to:

(A1) (translation invariance) $\mathcal{T}_{y} Q=Q \mathcal{T}_{y}$ for $y \in \mathbb{R}$;

(A2) (continuity) $Q: \mathcal{C}_{b} \longrightarrow \mathcal{C}_{b}$ is continuous with respect to the compact open topology;

(A3) (compactness) the set $\left\{Q[u](x) \mid u \in \mathcal{C}_{b}, x \in \mathbb{R}\right\} \subset \mathcal{X}$ is precompact;

(A4) (monotonicity) $Q: \mathcal{C}_{b} \longrightarrow \mathcal{C}_{b}$ is monotone in the sense that $Q[u] \geq Q[v]$ whenever $u \geq v$ in $\mathcal{C}_{b}$

(A5) (monostability) $Q: \mathcal{X}_{b} \longrightarrow \mathcal{X}_{b}$ admits exactly two fixed points 0 and 1 and $\lim _{n \rightarrow \infty} Q^{n}[\bar{\omega}]=1$ for $\bar{\omega} \in \mathcal{X}_{b}$ and $\bar{\omega} \gg 0$.

Let $u(t, x ; \phi)$ be solution of (1.1) with initial value $\phi \in \mathcal{C}_{b}$. Define, $Q_{t}: \mathcal{C}_{b} \longrightarrow \mathcal{C}_{b}$ by

$$
Q_{t}[\phi](x)=u_{t}(x ; \phi)
$$

Lemma 2.6. $\left\{Q_{t}\right\}_{t \geq 0}$ is a semiflow on $\mathcal{C}_{b}$ with the time-one map $Q_{1}$ satisfying $(A 1)-(A 5)$.

\section{Proof.}

- To show that $\left\{Q_{t}\right\}_{t \geq 0}$ is a semiflow on $\mathcal{C}_{b}$ we only need to prove that $Q_{t}[u]$ is continuous in $(t, u) \in[0, \infty) \times \mathcal{C}_{b}$ as properties $(i)$ and $(i i)$ are easily satisfied. A proof of the joint continuity can be found in [18] although we sketch it for completeness. Let $t, t_{n} \in[0, \tau)$ and $\phi, \phi_{n} \in \mathcal{C}_{b}$ with $t_{n} \rightarrow t$ and $\phi_{n} \rightarrow \phi$. When $t_{n}+\theta \leq 0$, we have that

$$
\left|Q_{t_{n}}\left[\phi_{n}\right](\theta, x)-Q_{t_{n}}[\phi](\theta, x)\right|=\left|\phi_{n}\left(t_{n}+\theta, x\right)-\phi\left(t_{n}+\theta, x\right)\right| \leq \max _{s \in \Omega}\left|\phi_{n}(s, x)-\phi(s, x)\right|
$$

whereas when $t_{n}+\theta \geq 0$,

$\left|Q_{t_{n}}\left[\phi_{n}\right](\theta, x)-Q_{t_{n}}[\phi](\theta, x)\right| \leq\left|\phi_{n}(0, x)-\phi(0, x)\right|+s_{m} \int_{\mathbb{R}} \mathcal{K}(x-y) \max _{s \in \Omega}\left|\phi_{n}(s, y)-\phi(s, y)\right| \mathrm{d} y$.

Since $\mathcal{K} \in L^{1}(\mathbb{R})$ and $\phi_{n} \rightarrow \phi$, for $\epsilon>0$ and $[a, b] \subset \mathbb{R}$, we can find $N_{1}, N_{2}>0$ such that

$$
s_{m} \int_{\mathbb{R} \backslash\left[-N_{1}, N_{1}\right]} \mathcal{K}(y) \mathrm{d} y<\epsilon / 3
$$

and for $n \geq N_{2}$,

$$
\max _{x \in[a, b]}\left|\phi_{n}(0, x)-\phi(0, x)\right|<\epsilon / 3 \quad \text { and } \max _{s \in \Omega, x \in\left[a-N_{1}, b+N_{1}\right]}\left|\phi_{n}(s, x)-\phi(s, x)\right|<\epsilon /\left(3 s_{m}\right) .
$$

As a consequence, for $n \geq N_{2}$ we have

$$
\max _{s \in \Omega, x \in[a, b]}\left|Q_{t_{n}}\left[\phi_{n}\right](\theta, x)-Q_{t_{n}}[\phi](\theta, x)\right|<\epsilon / 3,
$$

and $\left\|Q_{t_{n}}\left[\phi_{n}\right]-Q_{t_{n}}[\phi]\right\| \rightarrow 0$ as $n \rightarrow \infty$. By a similar argument we can show that $\| Q_{t_{n}}[\phi]-$ $Q_{t}[\phi] \| \rightarrow 0$ as $n \rightarrow \infty$ and the triangular inequality concludes the proof. 
- We now turn to the properties (A1)-(A5) for the time-map $Q_{1}$. The continuity (A2) and the monotony (A4) are automatically satisfied. The translation invariance (A1) follows from the uniqueness of solutions of (1.1). Note that the restriction of $\left\{Q_{t}\right\}_{t \geq 0}$ on $\mathcal{X}_{b}$ is the solution semiflow of the kinetic system

$$
\frac{\mathrm{d}}{\mathrm{d} t} u(t)=-u(t)+S(u(t-\tau))
$$

which is a delayed differential equation. It is well a known result (see [35]) that in that case, for initial condition $\phi \in \mathcal{X}_{b}$ with $\phi \gg 0$, the solution of (2.6) converges to $u=1$ and (A5) holds. Finally, without loss of generality, we can assume that $\tau<1$. Otherwise, we do a time rescaling $s=t / \tau_{1}$ for a fixed $\tau_{1}>\tau$ and the consider the resulting equation on $v(s, x)=u\left(\tau_{1} s, x\right)$. Thus we have $1+\theta>0$ for $\theta \in[-\tau, 0]$, and $\partial_{\theta} Q_{1}[\phi](\theta, x)=\partial_{t} u(1+\theta, x ; \phi)$ is uniformly bounded in $x \in \mathbb{R}, \theta \in[-\tau, 0]$ and $\phi \in \mathcal{C}_{b}$. As a consequence, by Arzelà-Ascoli, $\left\{Q[u](x) \mid u \in \mathcal{C}_{b}, x \in \mathbb{R}\right\} \subset \mathcal{X}$ is precompact and (A4) holds.

We now present the definition of semiflow on the space $\mathcal{M}_{b}$ of monotone functions.

Definition 2.7. A family of mapping $\left\{Q_{t}\right\}_{t \geq 0}$ is said to be a semiflow on $\mathcal{M}_{b}$ if the following properties hold:

(i) $Q_{0}=I$, where $I$ is the identity mapping;

(ii) $Q_{t} Q_{s}=Q_{t+s}$ for $t, s \geq 0$;

(iii) if $u_{n} \rightarrow u$ in $\mathcal{M}_{b}$ and $t_{n} \rightarrow t$, then both $Q_{t_{n}}[u](x) \rightarrow Q_{t}[u](x)$ and $Q_{t}\left[u_{n}\right](x) \rightarrow Q_{t}[u](x)$ in $\mathcal{X}$ almost everywhere.

The following assumptions on map $Q: \mathcal{M}_{b} \rightarrow \mathcal{M}_{b}$ will be referred to:

(B1) (translation invariance) $\mathcal{T}_{y} Q=Q \mathcal{T}_{y}$ for $y \in \mathbb{R}$;

(B2) (continuity) if $u_{n} \rightarrow u$ in $\mathcal{M}$, then $Q\left[u_{n}\right](x) \rightarrow Q_{t}[u](x)$ almost everywhere in $\mathcal{X}$;

(B3) (weak compactness) there exists $k \in[0,1)$ such that $\alpha(Q[\mathcal{U}](0)) \leq k \alpha(\mathcal{U}(0))$ for $\mathcal{U} \subset \mathcal{M}_{b}$;

(B4) (monotonicity) $Q: \mathcal{M}_{b} \longrightarrow \mathcal{M}_{b}$ is monotone in the sense that $Q[u] \geq Q[v]$ whenever $u \geq v$ in $\mathcal{M}_{b}$;

(B5) (monostability) $Q$ admits exactly two fixed points 0 and 1 in $\mathcal{X}_{+}$and $\lim _{n \rightarrow \infty} Q^{n}[\bar{\omega}]=1$ for $\bar{\omega} \in \mathcal{X}_{+}$and $0 \ll \bar{\omega} \leq 1$.

Lemma 2.8. The equation (1.1) generates a solution semiflow $\left\{Q_{t}\right\}_{t \geq 0}$ on $\mathcal{M}_{b}$ via (2.5) with each time-t maps $Q_{t}$ satisfying $(B 1)-(B 5)$ for $t>0$. 
Proof. From Lemma 2.1 and 2.6, we directly see that $\left\{Q_{t}\right\}_{t \geq 0}$ defines a semiflow on $\mathcal{M}_{b}$. Furthermore, it is easy to check that each time- $t$ map $Q_{t}$ satisfies (B1), (B2), (B4) and (B5) with $Q=Q_{t}$ and fixed points replaced by equilibria of the semiflow in (B5). Then, one needs only to show (B3). Following the same decomposition arguments as in [17, Theorem 5.2], we obtain that $\alpha\left(Q_{t}[\mathcal{U}](0)\right) \leq e^{-\gamma t} \alpha(\mathcal{U}(0)), \mathcal{U} \subset \mathcal{M}_{b}$ for some $\gamma>0$.

\subsection{The case $\tau=0$}

Throughout this section, we have implicitly assumed that $\tau>0$. The case $\tau=0$ is actually slightly different to handle and we treat it here separately. We consider the Cauchy problem,

$$
\left\{\begin{aligned}
\partial_{t} u(t, x) & =-u(t, x)+\int_{\mathbb{R}} \mathcal{K}(x-y) S(u(t, y)) \mathrm{d} y & & \text { for }(t, x) \in(0, \infty) \times \mathbb{R}, \\
u(0, x) & =\phi(x) & & \text { for } x \in \mathbb{R} .
\end{aligned}\right.
$$

We define $\mathcal{Y}=\{u \in C(\mathbb{R}, \mathbb{R}) \mid 0 \leq u \leq 1\}$ and $\mathcal{Z}=\{u \in C(\mathbb{R}, \mathbb{R}) \mid 0 \leq u \leq 1, u$ monotone $\} \subset \mathcal{Y}$.

Lemma 2.9. For an initial condition $\phi \in \mathcal{Y}$ (respectively $\mathcal{Z}$ ), equation (2.7) admits a unique solution $(t, x) \mapsto u(t, x ; \phi)$ which exists globally in time $t \geq 0$, that is $t \mapsto u(t, \cdot ; \phi) \in C^{1}([0 ;+\infty), C(\mathbb{R}, \mathbb{R}))$, such that $u(0, \cdot)=\phi$ and $u(t, \cdot) \in \mathcal{X}$ (respectively $\mathcal{Z}$ ) for $t \geq 0$. Furthermore, the comparison principle of Lemma 2.4 still holds true.

Proof. We refer to [37] for the existence, uniqueness of solutions and the comparison principle.

Let $u(t, x ; \phi)$ be a solution of the Cauchy problem $(2.7)$ with initial value $\phi \in \mathcal{Y}$. Define, $Q_{t}: \mathcal{Y} \rightarrow \mathcal{Y}$ by

$$
Q_{t}[\phi](x)=u(t, x ; \phi) .
$$

Lemma 2.10. We have the following properties for the flow $\left\{Q_{t}\right\}_{t \geq 0}$ :

(i) $\left\{Q_{t}\right\}_{t \geq 0}$ is a semiflow on $\mathcal{Y}$ with the time-one map $Q_{1}$ satisfying $(A 1)-(A 5)$;

(ii) $\left\{Q_{t}\right\}_{t \geq 0}$ is a semiflow on $\mathcal{Z}$ with each time-t map $Q_{t}$ satisfying $(B 1)-(B 5)$ for $t>0$.

Proof. To show that $\left\{Q_{t}\right\}_{t \geq 0}$ is a semiflow on $\mathcal{Y}$ we only need to prove that $Q_{t}[u]$ is continuous in $(t, u) \in[0, \infty) \times \mathcal{Y}$ as properties $(i)$ and $(i i)$ are obviously satisfied. Let $t, t_{n} \geq 0$ and $\phi, \phi_{n} \in \mathcal{Y}$ with $t_{n} \rightarrow t$ and $\phi_{n} \rightarrow \phi$. Using the variation of constant formula, we see that

$$
Q_{t}[\phi](x)=e^{-t} \phi(x)+\int_{0}^{t} e^{-(t-s)}\left(\int_{\mathbb{R}} \mathcal{K}(x-y) S\left(Q_{s}[\phi](y)\right) \mathrm{d} y\right) \mathrm{d} s .
$$

As a consequence, if we denote $w_{n}(t, x):=\left|Q_{t}\left[\phi_{n}\right](x)-Q_{t}[\phi](x)\right|$, then we have

$$
w_{n}(t, x) \leq e^{-t} w_{n}(0, x)+s_{m} \int_{0}^{t} e^{-(t-s)}\left(\int_{\mathbb{R}} \mathcal{K}(x-y) w_{n}(s, y) \mathrm{d} y\right) \mathrm{d} s .
$$


Let $v_{n}(t, x)$ be the solution to the following integral problem

$$
\left\{\begin{aligned}
& v_{n}(t, x)=e^{-t} v_{n}(0, x)+s_{m} \int_{0}^{t} e^{-(t-s)} \mathcal{K} * v_{n}(s, x) \mathrm{d} s, \quad(t, x) \in(0, \infty) \times \mathbb{R}, \\
& v_{n}(0, x)=w_{n}(0, x), \\
& x \in \mathbb{R}
\end{aligned}\right.
$$

where $\mathcal{K} * v(x):=\int_{\mathbb{R}} \mathcal{K}(x-y) v(y) \mathrm{d} y$. Using the comparison principle, it is straightforward to check that $w_{n}(t, x) \leq v_{n}(t, x)$ for all $(t, x) \in(0, \infty) \times \mathbb{R}$. Also, a direct computation shows that $v_{n}(t, x)$ satisfies the equation

$$
\partial_{t} v_{n}(t, x)=-v_{n}(t, x)+s_{m} K * v_{n}(t, x) .
$$

It then follows from [45, Lemma 3.1] that $v_{n}$ has the following representation formula

$$
v_{n}(t, x)=e^{-t} \sum_{m=0}^{\infty} \frac{\left(s_{m} t\right)^{m}}{m !} \mathcal{K}_{m} * v_{n}(0, x)
$$

where we set the following notation

$$
\mathcal{K}_{m}:= \begin{cases}\underbrace{\mathcal{K} * \cdots * \mathcal{K}}_{\begin{array}{l}
m \text { times } \\
\text { Dirac measure },
\end{array}}, & m \geq 0 .\end{cases}
$$

Using the fact that $\mathcal{K}_{m} * v_{n}(0, x) \rightarrow 0$ as $n \rightarrow \infty$ on compact set, we can then use similar arguments as in the proof of Lemma 2.6 to conclude that $Q_{t_{n}}\left[\phi_{n}\right] \rightarrow Q_{t}[\phi]$ as $n \rightarrow 0$. It is then straightforward to check that the time-one map $Q_{1}$ satisfies $(A 1)-(A 5)$ and that each time- $t$ map $Q_{t}$ satisfies $(B 1)-(B 5)$ for $t>0$.

\subsection{Proofs of Theorems 2 and 3}

We are now in position to prove Theorems 2 and 3 using dynamical systems theories developed recently in $[17,27,28]$.

\subsubsection{Spreading speeds}

The following lemma is a combination of [27, Theorems 2.11, 2.15 and 2.17] and [28, Theorems 3.1-3.14]. A map $Q: \mathcal{X}_{b} \rightarrow \mathcal{X}_{b}$ is said to be sub-homogeneous if $Q[\rho \phi] \geq \rho Q[\phi]$ for all $\rho \in[0,1]$ and $\phi \in \mathcal{X}_{b}$.

Lemma 2.11 (see $[27,28]$ ). Assume that $\left\{Q_{t}\right\}_{t \geq 0}$ is a semiflow on $\mathcal{X}_{b}$ and $Q=Q_{1}$ satisfies all hypotheses (A1)-(A5). Then the semiflow admits leftward/rightward spreading speeds $c_{ \pm}^{*}$ in the following sense:

(i) if $c>c_{+}^{*}$ and $c^{\prime}>c_{-} *$ and the initial condition $\phi$ has compact support with $0 \leq \phi \ll \omega \ll 1$, with $\omega \in \mathcal{X}$ then

$$
\lim _{t \rightarrow \infty, x \geq c t} Q_{t}[\phi](x)=0 \text { and } \lim _{t \rightarrow \infty, x \leq-c^{\prime} t} Q_{t}[\phi](x)=0 \quad \text { in } \in \mathcal{X}
$$


(ii) assume that $c_{+}^{*}+c_{-}^{*}>0$. If $c<c_{+}^{*}$ and $c^{\prime}<c_{-}^{*}$ with $c+c^{\prime}>0$ and $\sigma \in \mathcal{X}$ with $\sigma \gg 0$, there is a positive number $\ell_{\sigma}>0$ such that if the initial condition $\phi \in \mathcal{C}_{b}$ satisfies $\phi(x) \geq \sigma$ for $x$ on an interval of length $2 \ell_{\sigma}$, then

$$
\lim _{t \rightarrow \infty, c^{\prime} t \leq x \leq c t} Q_{t}[\phi](x)=1 .
$$

In addition, if $Q_{1}$ is sub-homogeneous, then $\ell_{\sigma}$ can be chosen independent of $\sigma$.

\section{Proof. [Proof of Theorem 2]}

- From Lemma 2.6 and 2.11, we have the existence of leftward/rightward spreading speeds $c_{ \pm}^{*}$ in the sense of Lemma 2.11. We will first show that $c_{+}^{*}+c_{-}^{*}>0$. By the assumption (H2), we can find a $\mathcal{C}^{1}$ function $S_{1}$ with

$$
S_{1}(u) \leq S(u), \quad S_{1}^{\prime}(0)>1, \quad S_{1}(u) \leq S_{1}^{\prime}(0) u \quad u \in[0,1] .
$$

Finally, let $A>0$ and define $\mathcal{K}^{A}$ by

$$
\mathcal{K}^{A}(x)=\left\{\begin{array}{cc}
\mathcal{K}(x) & x \in[-A, A] \\
0 & \text { otherwise }
\end{array}\right.
$$

We can then consider the following auxiliary equation

$$
\partial_{t} u(t, x)=-u(t, x)+\int_{\mathbb{R}} \mathcal{K}^{A}(x-y) S_{1}(u(t-\tau, y)) \mathrm{d} y, \quad(t, x) \in(0, \infty) \times \mathbb{R} .
$$

This system trivially admits leftward/rightward spreading speeds $c_{ \pm}(A)$ in the sense of Lemma 2.11 which satisfies $c_{+}^{*}+c_{-}^{*}>c_{+}(A)+c_{-}(A)$ for any $A>0$ sufficiently large to ensure that equation (2.9) is still of monostable type. Following the general approach presented in $[27$, section 3], we can obtain that $c_{ \pm}(A)=\min _{\mu>0} \frac{\lambda_{1}( \pm \mu)}{\mu}$, where $\lambda_{1}(\mu)$ is the principal eigenvalue of the linearization at zero state for the delayed equation (2.9), that is, $\lambda$ is the unique real solution of

$$
\lambda=-1+S_{1}^{\prime}(0) e^{-\lambda \tau} \int_{\mathbb{R}} e^{\mu x} \mathcal{K}^{A}(x) \mathrm{d} x .
$$

We refer to [35] for more descriptions on principle eigenvalues of delayed equations. Note that the integral on the right-hand side of the equality is well-defined because of the definition of $\mathcal{K}^{A}$. Suppose that the minimum values of $\frac{\lambda_{1}( \pm \mu)}{\mu}$ are attained at $\mu_{1}>0$ and $\mu_{2}>0$ respectively, then by the convexity of the principal eigenvalue (see [27, section 3]), we have that

$$
\begin{aligned}
c_{+}(A)+c_{-}(A) & =\frac{\lambda_{1}\left(-\mu_{1}\right)}{\mu_{1}}+\frac{\lambda_{1}\left(\mu_{2}\right)}{\mu_{2}}=\frac{\mu_{1}+\mu_{2}}{\mu_{1} \mu_{2}}\left(\frac{\mu_{2}}{\mu_{1}+\mu_{2}} \lambda_{1}\left(-\mu_{1}\right)+\frac{\mu_{1}}{\mu_{1}+\mu_{2}} \lambda_{1}\left(\mu_{2}\right)\right) \\
& \geq \frac{\mu_{1}+\mu_{2}}{\mu_{1} \mu_{2}} \lambda_{1}(0)>0,
\end{aligned}
$$

since $S_{1}^{\prime}(0)>1$. 
- We still need to weaken condition (ii) of Lemma 2.11 to be $\phi \not \equiv 0$ to conclude the proof. To do so, let $u_{*}$ be the smallest positive solution of the equation $-u+S_{1}(u)=0$. We can chose $\epsilon_{0}>0$ such that for any initial condition $\phi \in \mathcal{C}_{b}$ we have $\epsilon_{0} \phi \leq u_{*}$. As a consequence, it is shown in [18, Lemma 4.4] that if $\phi \not \equiv 0$, then the solution $Q_{t}\left[\epsilon_{0} \phi\right](x)>0$ of (2.9) for all $t \geq \tau$ and $x \in \mathbb{R}$. Furthermore, note that $Q_{1}$ is sub-homogenous so that we can chose $\ell_{\sigma}$ in Lemma 2.11 independent of $\sigma$ and then define

$$
\sigma:=\min _{(\theta, x) \in[-\tau, 0] \times[-\ell, \ell]} u\left(1+\theta, x ; \epsilon_{0} \phi\right)>0 .
$$

As a consequence, for $c_{1}<c_{+}(A)$ and $c_{2}<c_{-}(A)$ with $c_{1}+c_{2}>0$, we have

$$
\lim _{t \rightarrow \infty} \inf _{-t c_{2} \leq x \leq t c_{1}} Q_{t}\left[Q_{1}\left[\epsilon_{0} \phi\right]\right](x)=\lim _{t \rightarrow \infty} \inf _{-(t-1) t c_{2} \leq x \leq(t-1) c_{1}} Q_{t}\left[\epsilon_{0} \phi\right](x)=u_{*},
$$

then for any $K>0$, there exists $t_{0}>0$ such that $\left(c_{1}+c_{2}\right)\left(t_{0}-1\right) \geq K$ and

$$
\min _{-(t-1) c_{2} \leq x \leq(t-1) c_{1}} u(t, x ; \phi) \geq \frac{u_{*}}{2}, \quad \text { for } t \geq t_{0} .
$$

As conclusion, we have weakened condition (ii) of Lemma 2.11 to be $\phi \not \equiv 0$ and this concludes the proof of Theorem 2 .

\subsubsection{Monotone traveling fronts}

Regarding the existence of monotone traveling fronts, we cannot readily apply the results of [27, 28], as there is a lack of compactness in equation (1.7) due to the absence of random diffusion. Instead, we will use the recently developed theory of Fang and Zhao of monotone semiflows with weak compactness [17]. The following lemma is a combination of [17, Theorems 4.2 and Remark 3.7].

Lemma 2.12. Let $c_{ \pm}^{*}$ be the invasion speeds associated with Lemma 2.11 for the semiflow $\left\{Q_{t}\right\}_{t \geq 0}$ on $\mathcal{X}_{b}$. Assume that $\left\{Q_{t}\right\}_{t \geq 0}$ is semiflow on $\mathcal{M}_{b}$ and for $t>\tau$ the maps $Q_{t}$ satisfies all hypotheses (B1)-(B5). Then $c_{ \pm}^{*}$ are the minimal wave speeds for the rightward/leftward traveling waves.

Proof. [Proof of Theorem 3] The nonexistence of leftward/rightward monotone traveling waves with speed less than $c_{-}^{*} / c_{+}^{*}$ follows directly from the comparison principle and the property of invasion speed. For the existence, it is enough to show that solutions of (1.7) generate a semiflow on $\mathcal{M}_{b}$ which satisfies all conditions $(B 1)-(B 5)$. These are precisely the results of Lemma 2.8 in the case $\tau>0$ and Lemma 2.10 in the non-delayed case.

\section{Characterization of the minimal wave speed - Exponentially bounded kernels}

Throughout this section, we suppose that the Hypothesis (H1) on the connectivity kernel $\mathcal{K}$ holds and we further assume that $\mathcal{K}$ is symmetric. In that case, following Remark 1.1, we have that 
$c_{-}^{*}=c_{+}^{*}:=c^{*} \in(-\infty ;+\infty]$.

\subsection{Proof of Theorem 4}

We say that a kernel $\mathcal{K}$ is exponentially bounded if there exists $\mu_{0} \in(0, \infty]$ such that

$$
\int_{\mathbb{R}} \mathcal{K}(x) e^{\mu|x|} \mathrm{d} x<\infty, \quad \forall 0 \leq|\mu|<\mu_{0} \text { with } \lim _{\mu \uparrow \mu_{0}} \int_{\mathbb{R}} \mathcal{K}(x) e^{\mu|x|} \mathrm{d} x=+\infty .
$$

Examples of such kernels are Gaussian kernels of the form $\mathcal{K}(x)=e^{-\alpha x^{2}} / \sqrt{\pi \alpha}$ for $\alpha>0$ for which condition (3.1) is satisfied for all $\mu_{0}>0$ and exponential kernels of the form $\mathcal{K}(x)=e^{-\alpha|x|} / 2 \alpha$ for $\alpha>0$ for which (3.1) is satisfied with $\mu_{0}=\alpha$.

In order to simplify the presentation and to obtain closed form formula for the minimal wave speed in the case of exponentially bounded kernels, we make the additional assumption that the nonlinearity satisfies the so-called KPP assumption. That is we assume

$$
S \in C^{2}([0,1]) \text { is concave }, \quad S(0)=0, \quad S(1)=1, \quad \text { and } 1<S^{\prime}(0) .
$$

With these extra assumptions on the nonlinearity $S$ we obtain the following results on the characterization of the minimal wave speed.

Lemma 3.1. Suppose that the kernel $\mathcal{K}$ is exponentially bounded satisfying (3.1) for a given $\mu_{0}>0$. Furthermore, suppose that the nonlinearity S satisfies Hypothesis (H2) and is chosen such that (3.2) is satisfied. Then, the minimal wave speed $c^{*}(\tau)$ given in Theorem 2 satisfies

$$
0<c^{*}(\tau):=\min _{0<\mu<\mu_{0}} \frac{\lambda^{\tau}(\mu)}{\mu}<\infty
$$

where $\lambda^{\tau}(\mu)>0$ is the principal eigenavlue of

$$
\lambda=-1+S^{\prime}(0) e^{-\lambda \tau} \widetilde{\mathcal{K}}(\mu),
$$

with $\widetilde{\mathcal{K}}(\mu):=\int_{\mathbb{R}} \mathcal{K}(x) e^{\mu x} \mathrm{~d} x$. Furthermore, we have that $\tau \mapsto c^{*}(\tau)$ is continuous on $[0,+\infty)$, monotone decreasing with asymptotic behavior

$$
\lim _{\tau \rightarrow+\infty} c^{*}(\tau)=0
$$

Proof. Using the results of Liang and Zhao [27] together with the ones of Weinberger \& Zhao [44] and the fact that $S(u) \leq S^{\prime}(0) u$ with $S^{\prime}(0)>1$, we have the linear characterization of the wave speed as stated in (3.3). Using the definition of the principal eigenvalue and the fact that for all $\tau>0$ and all $0<\mu<\mu_{0}$ we have $\tau e^{\tau} S^{\prime}(0) \widetilde{\mathcal{K}}(\mu)>0$, we verify that

$$
\lambda^{\tau}(\mu)=\frac{1}{\tau} W_{0}\left(\tau e^{\tau} S^{\prime}(0) \widetilde{\mathcal{K}}(\mu)\right)-1,
$$


where $W_{0}$ is the principal branch of the Lambert function which satisfies $W_{0}(x) e^{W_{0}(x)}=x$ for any $x \geq 0$. As a consequence, we have

$$
c^{*}(\tau)=\min _{0<\mu<\mu_{0}}\left\{\frac{1}{\tau \mu}\left(W_{0}\left(\tau e^{\tau} S^{\prime}(0) \widetilde{\mathcal{K}}(\mu)\right)-\tau\right)\right\} .
$$

From the explicit form of the principal eigenvalue $\lambda^{\tau}(\mu)$ in (3.4), we can conclude that for every fixed $0<\mu<\mu_{0}$, the function $\tau \rightarrow \lambda^{\tau}(\mu)$ is monotonically decreasing toward zero, properties that are inherited by the function $\tau \rightarrow c^{*}(\tau)$.

Remark 3.2. It is important to note that formulae of the type (3.4) have already been derived by Veltz in [42] in the general case of propagation axonal delays.

\subsection{Numerical illustration}

For example, if we choose the nonlinearity

$$
S(u):=u(2-u), \quad u \in[0,1],
$$

then $S$ satisfies the KPP assumptions (3.2) and the results of the above lemma apply. We illustrate these results in Figure 1 using this specific nonlinearity (3.5) for two different exponentially bounded kernels: $\mathcal{K}(x)=e^{-|x|} / 2$ and $\mathcal{K}(x)=e^{-x^{2}} / \sqrt{\pi}$ where we compare the exact formula for the minimal wave speed as given in (3.3) with direct numerical simulations for values of the delay $\tau \in[0,5]$. In Figure 1, we also clearly observe the monotony property of the wave speed as a function of the delay $\tau$. For each numerical simulation, we used the same initial condition:

$$
\phi(t, x)=u_{0}(x), \quad(t, x) \in[-\tau, 0] \times \mathbb{R},
$$

where $0 \leq u_{0} \leq 1\left(u_{0} \not \equiv 0\right)$ is a continuous compactly supported perturbation of a rectangle step function. For the spacial discretization, we have used $N=2^{15}$ grid points and Fast Fourrier Transform to treat the convolution kernel on a domain of size $[-200 \pi, 200 \pi]$. For the time discretization, we have used a forward Euler scheme with step size $\delta t=0.01$. Note that the error between theoretical prediction and numerical simulation for the wave speed is less than $4 \%$ and of the order $\mathcal{O}(\delta t)$.

\section{Uniqueness of traveling waves}

There exists a large literature regarding the uniqueness of monotone traveling wave solutions of equations of the form (1.2) going back to the pioneering work of Diekmann and Kaper [14]. Their original work was extended in several ways and we mention here the extensions of Carr and Chmaj [10] for nonlocal dispersal Fisher-KPP equation, of Fang and Zhao [16] for a class of integral equations including (1.1) and (1.4), and the more recent developments of Aguerrea et al. [1] which covers a more general class of integral equations. Our strategy to prove Theorem 5 will be to 


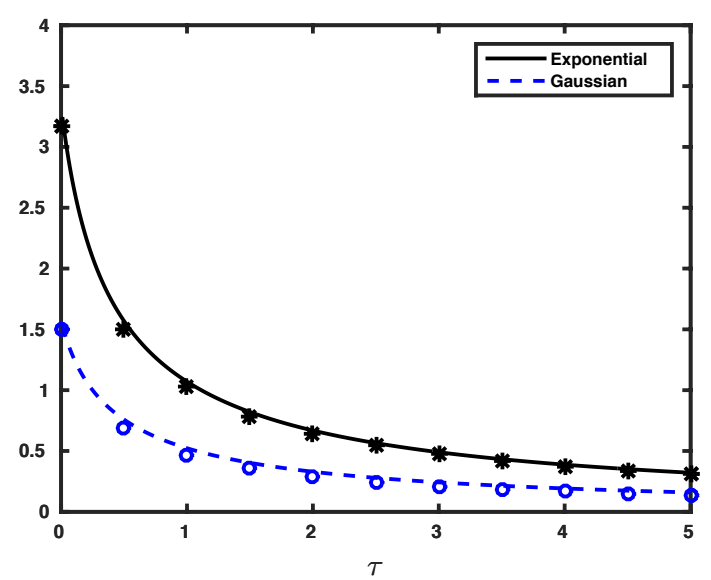

Figure 1: Comparison of the exact formula for the minimal wave speed as given in (3.3) with direct numerical simulations for the nonlinearity $S$ in (3.5) and two different exponentially bounded kernels. The black line corresponds to the theoretical wave speed prediction in the case of $\mathcal{K}(x)=$ $e^{-|x|} / 2$ and the stars are direct numerical simulations. The blue dashed line corresponds to the theoretical wave speed prediction in the case of $\mathcal{K}(x)=e^{-x^{2}} / \sqrt{\pi}$ and the circles are direct numerical simulations.

transform our traveling wave problem (1.2) and (1.3) into the integral formalism that has been developed in $[1,14,16]$.

Proof. [Proof of Theorem 5] By Theorem 3 and Remark 1.1 regarding symmetric kernel, we know there exists the minimal wave speed $c^{*} \in(-\infty,+\infty]$. Since $\mathcal{K}$ is exponentially bounded, it then follows from Theorem 4 that $c^{*}<+\infty$. Consequently, for each $c \geq c^{*}$ there exists a traveling wave $u(x-c t)$ with $u(+\infty)=0$ and $u(-\infty)=1$. Note that $u(\xi)$ satisfies $(1.2)$ which can be written as the following integral equation

$$
\frac{\mathrm{d}}{\mathrm{d} \xi}\left[u(\xi) e^{-\xi / c}\right]=-\frac{e^{-\xi / c}}{c} \mathcal{K} * S(u(\xi+c \tau)) .
$$

Integrating the previous equality from $(\xi,+\infty)$ using Fubini's theorem and the Lebesgue dominated convergence theorem together with the fact that $S(0)=0$, we obtain

$$
u(\xi)=\frac{1}{c} \int_{\xi}^{+\infty} e^{\left(\xi-\xi^{\prime}\right) / c} \mathcal{K} * S\left(u\left(\xi^{\prime}+c \tau\right)\right) \mathrm{d} \xi^{\prime} .
$$

We can write the above equation as

$$
u(\xi)=\mathcal{G}_{c}^{\tau} * S(u(\xi))
$$

where

$$
\mathcal{G}_{c}^{\tau}(\xi):=\frac{1}{c} \int_{\mathbb{R}} H\left(-\left(\xi-\xi^{\prime}\right)\right) e^{\left(\xi-\xi^{\prime}\right) / c} \mathcal{K}\left(\xi^{\prime}+c \tau\right) \mathrm{d} \xi^{\prime},
$$


and $H$ denotes the Heaviside step function. It is straightforward to check that $\mathcal{G}_{c}^{\tau}(\xi) \geq 0$ and is exponentially bounded for every $c \geq c^{*}$ and $\tau \geq 0$, with $\int_{\mathbb{R}} \mathcal{G}_{c}^{\tau}(\xi) \mathrm{d} \xi=1$. Since by hypothesis, $S$ is assumed to be $\mathcal{C}^{2}$ in a neighborhood of $u=0$, we can apply [1, Theorem 4] to obtain the uniqueness result for any $c \geq c^{*}$.

Finally, we further assume $S \in \mathcal{C}^{2}([0,1])$ and concave. Then, we can apply Lemma 3.1 to obtain the characterization of the minimal wave speed as

$$
0<c^{*}(\tau)=\min _{0<\mu<\mu_{0}} \frac{\lambda^{\tau}(\mu)}{\mu}=\frac{\lambda^{\tau}\left(\mu^{*}(\tau)\right)}{\mu^{*}(\tau)},
$$

for some $0<\mu^{*}(\tau)<\mu_{0}$ where $\lambda^{\tau}(\mu)>0$ is the principal eigenvalue of

$$
\lambda=-1+S^{\prime}(0) e^{-\lambda \tau} \widetilde{\mathcal{K}}(\mu) .
$$

From now on, in order to simplify the notations, we drop the dependence in $\tau$ and note $c^{*}=c^{*}(\tau)$ and $\mu^{*}=\mu^{*}(\tau)$. It can be easily shown [18], that $\left(\mu^{*}, c^{*}\right)$ is the unique solution to the $(\mu, c)$-system

$$
\mu>0, \quad F(\mu, c)=0, \quad \partial_{\mu} F(\mu, c)=0,
$$

where

$$
F(\mu, c):=c \mu+1-S^{\prime}(0) e^{-\mu c \tau} \widetilde{\mathcal{K}}(\mu) .
$$

Also, note that the function $\Psi^{\tau}(\mu)=\lambda^{\tau}(\mu) / \mu$ is positive for all $\mu>0$. Furthermore, at $\mu=\mu^{*}$, we have

$$
\frac{\mathrm{d}}{\mathrm{d} \mu} \Psi^{\tau}\left(\mu^{*}\right)=0 \text { and } \frac{\mathrm{d}^{2}}{\mathrm{~d} \mu^{2}} \Psi^{\tau}\left(\mu^{*}\right)>0 .
$$

As a conclusion, for every $c>c^{*}$ the equation $F(\mu, c)=0$ has two positive roots: $0<\mu_{1}(c)<$ $\mu_{2}(c)<\mu_{0}$, and a double root $\mu^{*}$ for $c=c^{*}$. Then we apply [1, Theorem 3] to obtain the asymptotic behavior.

\section{$5 \quad$ Exponentially unbounded kernels}

We say that a kernel $\mathcal{K}$ is exponentially unbounded if $\mathcal{K}$ is a $\mathcal{C}^{1}$ function for large $x$, and

$$
\mathcal{K}^{\prime}(x)=o(\mathcal{K}(x)) \quad \text { as } \quad|x| \rightarrow+\infty .
$$

This condition implies that $\mathcal{K}$ decays more slowly than any exponentially decaying functions, in the sense that

$$
\mathcal{K}(x) e^{\mu|x|} \rightarrow+\infty \quad \text { as } \quad|x| \rightarrow+\infty
$$

for all $\mu>0$. 


\subsection{Acceleration phenomenon}

We first show that acceleration occurs in the case of exponentially unbounded kernels.

Lemma 5.1. Suppose that the kernel $\mathcal{K}$ is exponentially unbounded satisfying (5.1). Assume that the nonlinearity $S$ satisfies Hypothesis (H2). Then, the minimal wave speed satisfies

$$
c^{*}(\tau)=+\infty,
$$

for all $\tau \geq 0$.

Proof. The argument is standard. By the assumption (H2), we can find a $\mathcal{C}^{1}$ function $S_{1}$ with

$$
S_{1}(u) \leq S(u), \quad S_{1}^{\prime}(0)>1, \quad S_{1}(u) \leq S_{1}^{\prime}(0) u \quad u \in[0,1] .
$$

Let $A>0$ and define $\mathcal{K}^{A}$ by

$$
\mathcal{K}^{A}(x)=\left\{\begin{array}{cc}
\mathcal{K}(x) & x \in[-A, A], \\
0 & \text { otherwise } .
\end{array}\right.
$$

Once again, we can then consider the following auxiliary equation

$$
\partial_{t} u(t, x)=-u(t, x)+\int_{\mathbb{R}} \mathcal{K}^{A}(x-y) S_{1}(u(t-\tau, y)) \mathrm{d} y, \quad(t, x) \in(0, \infty) \times \mathbb{R},
$$

for $A>0$ sufficiently large to ensure that this equation is of monostable type for which we can apply the results on exponentially bounded kernels (see Liang and Zhao [27]) and find that the minimal wave speed $\underline{\mathrm{c}}_{A}(\tau)$ is explicitly given by

$$
\underline{\mathrm{c}}_{A}(\tau)=\min _{\mu>0}\left\{\frac{1}{\tau \mu}\left(W_{0}\left(\tau e^{\tau} S_{1}^{\prime}(0) \widetilde{\mathcal{K}^{A}}(\mu)\right)-\tau\right)\right\}=\frac{\lambda^{\tau}\left(\mu_{A}\right)}{\mu_{A}},
$$

for some $\mu_{A}>0$, and where $\lambda^{\tau}(\mu)=W_{0}\left(\tau e^{\tau} S_{1}^{\prime}(0) \widetilde{\mathcal{K}^{A}}(\mu)\right) / \tau-1$. There are three possibilities for the behavior of the sequence $\left(\mu_{A}\right)_{A \geq 0}$ : either it is a bounded sequence, or it has a subsequence converging to 0 or a subsequence diverging to $+\infty$. Since $\lambda^{\tau}\left(\mu_{A}\right) \rightarrow+\infty$ as $A \rightarrow \infty$, it remains to consider the second and third possibilities. Fix $A>1$, then we have $\frac{\lambda^{\tau}(\mu)}{\mu} \rightarrow+\infty$ as $\mu \rightarrow 0$ or as $\mu \rightarrow+\infty$. This, together with the monotonicity of $\lambda^{\tau}(\mu)$ in $A$, implies $\underline{\mathrm{c}}_{A}(\tau) \rightarrow+\infty$ as $A \rightarrow+\infty$ in all cases. Finally, it is enough to notice that the minimal wave speed $c^{*}(\tau)$ is bounded by below by $\underline{\mathrm{c}}_{A}(\tau)$ for any $\tau \geq 0$ and any $A>0$ to conclude the proof.

\subsection{Case study - Algebraically decaying kernels}

In this subsection, we discuss in more details the location of the level sets associated to the solutions of the Cauchy problem (1.7) in the case of algebraically decaying kernels which are a particular case of exponentially unbounded kernels. From now on, we suppose that $\mathcal{K}$ is given by

$$
\mathcal{K}(x)=\frac{C_{\alpha}}{1+|x|^{2 \alpha}}, \quad \alpha>1 / 2, \text { and } \quad C_{\alpha}:=\left(\int_{\mathbb{R}} \frac{1}{1+|x|^{2 \alpha}} \mathrm{d} x\right)^{-1} .
$$


It is of course straightforward to check that Hypothesis (H1) and (5.1) are satisfied for such kernels. For any $\tau \geq 0$ and any $\kappa \in(0,1)$ and $t \geq 0$, we denote by

$$
E_{\kappa}^{\tau}(t)=\{x \in \mathbb{R} \mid u(t, x)=\kappa\},
$$

for any $u$, solution of (1.7) with a continuous and compactly supported initial condition $\phi \in \mathcal{X}_{b}$. Since $u(t, x)$ spreads towards the positive state eventually faster than any finite speed as shown in Lemma 5.1, we have $u(t, x)>\kappa$ uniformly for any $x$ in bounded set as $t \rightarrow \infty$. Meanwhile, for any $t$, we can show that $u(t, x) \rightarrow 0$ as $|x| \rightarrow \infty$ thanks to the explicit expression of $u$. This, together with the continuity of $u(t, x)$, implies that $E_{\kappa}^{\tau}(t) \neq \emptyset$. As a consequence, we also have $\sup _{x \in E_{\kappa}^{\tau}(t)}|x|>\gamma t$ for all $\gamma>0$ as long as $t$ is sufficiently large. Next, we give an upper bound for $E_{\kappa}^{\tau}(t)$.

\subsubsection{An upper bound}

We first start with an upper bound for the location of the level set $E_{\kappa}^{\tau}(t)$. Let define

$$
r:=\sup _{u \in(0,1]} \frac{S(u)}{u} \geq S^{\prime}(0)>1 .
$$

A direct computation [23] shows that there exists $M_{\alpha} \geq 1$ such that for all $x \in \mathbb{R}$,

$$
\int_{\mathbb{R}} \mathcal{K}(x-y) \frac{1}{1+|y|^{2 \alpha}} \mathrm{d} y \leq \frac{M_{\alpha}}{1+|x|^{2 \alpha}} .
$$

Finally, let us define $\bar{\rho}(\tau)>0$ as the unique positive root of

$$
\rho+1-r e^{-\rho \tau} M_{\alpha}=0 .
$$

Proposition 5.2. Let $u$ be the solution of (1.7) with a continuous and compactly supported initial condition $\phi \in \mathcal{X}_{b}(\phi \neq \equiv 0)$. Assume that $\mathcal{K}$ is given by (5.3) and that the nonlinearity $S$ satisfies Hypothesis (H2). Then, for any $\tau \geq 0$ any $\kappa \in(0,1)$, we have

$$
\limsup _{t \rightarrow \infty} \frac{\log (\max \{x: u(t, x)=\kappa\})}{t} \leq \frac{\bar{\rho}(\tau)}{2 \alpha} .
$$

Proof. For the proof of the proposition, we follow the strategy developed by Garnier [23] and adapt it to our setting. The idea is to construct a super solution with the same asymptotic behavior as the kernel $\mathcal{K}$. More precisely, let define

$$
\bar{u}(t, x)=\min \left\{\frac{\eta}{1+|x|^{2 \alpha}} e^{\bar{\rho}(\tau) t}, 1\right\},
$$

where $\bar{\rho}(\tau)>0$ is given in (5.4) and $\eta>0$ chosen such that

$$
\phi(t, x) \leq \bar{u}(0, x) \text { for all }(t, x) \in[-\tau, 0] \times \mathbb{R} .
$$


Let also define

$$
N(\bar{u}(t, x)):=\partial_{t} \bar{u}(t, x)+\bar{u}(t, x)-\int_{\mathbb{R}} \mathcal{K}(x-y) S(\bar{u}(t-\tau, y)) \mathrm{d} y,
$$

for all $(t, x) \in[-\tau, 0] \times \mathbb{R}$. It is a straightforward computation to check that where $\bar{u}(t, x)=1$, we have

$$
N(\bar{u}(t, x)) \geq 1-\int_{\mathbb{R}} \mathcal{K}(x-y) S(\bar{u}(t-\tau, y)) \mathrm{d} y \geq 0 .
$$

In the region where $\bar{u}(t, x)<1$, we have that

$$
N(\bar{u}(t, x)) \geq(\bar{\rho}(\tau)+1) \frac{\eta}{1+|x|^{2 \alpha}} e^{\bar{\rho}(\tau) t}-r \int_{\mathbb{R}} \mathcal{K}(x-y) \bar{u}(t-\tau, y) \mathrm{d} y,
$$

where we used the definition of $r>0$. Finally, we have that

$$
\int_{\mathbb{R}} \mathcal{K}(x-y) \bar{u}(t-\tau, y) \mathrm{d} y \leq \eta e^{\bar{\rho}(\tau)(t-\tau)} \int_{\mathbb{R}} \mathcal{K}(x-y) \frac{1}{1+|y|^{2 \alpha}} \mathrm{d} y \leq M_{\alpha} e^{-\bar{\rho}(\tau) \tau} \frac{\eta}{1+|x|^{2 \alpha}} e^{\bar{\rho}(\tau) t} .
$$

As a consequence, using the definition of $\bar{\rho}(\tau)>0$, in the region where $\bar{u}(t, x)<1$, we have that

$$
N(\bar{u}(t, x)) \geq\left(\bar{\rho}(\tau)+1-r e^{-\bar{\rho}(\tau) \tau} M_{\alpha}\right) \bar{u}(t, x)=0 .
$$

This shows that $\bar{u}(t, x)$ is a super solution for any $t \geq 0$ and $x \in \mathbb{R}$ with $\phi(t, x) \leq \bar{u}(0, x)$ for all $(t, x) \in[-\tau, 0] \times \mathbb{R}$ such that, by the comparison principle, we have

$$
u(t, x) \leq \bar{u}(t, x) \quad(t, x) \in(0, \infty) \times \mathbb{R} .
$$

From this inequality and the definition of $\bar{u}(t, x)$, we easily deduce that there exists $t_{\kappa}^{\tau}>0$ such that for all $t \geq t_{\kappa}^{\tau} E_{\kappa}^{\tau}(t)$ is not empty and thus

$$
\limsup _{t \rightarrow \infty} \frac{\log (\max \{x: u(t, x)=\kappa\})}{t} \leq \frac{\bar{\rho}(\tau)}{2 \alpha} .
$$

\subsubsection{A lower bound}

We know provide a lower bound for the location of the level set $E_{\kappa}^{\tau}(t)$. First we define $\rho^{*}(\tau)>0$ as the unique positive solution of

$$
\rho+1-S^{\prime}(0) e^{-\rho \tau}=0 .
$$

It is worth noticing to note that we always have $\rho^{*}(\tau) \leq \bar{\rho}(\tau)$ for all $\tau \geq 0$ as $r M_{\alpha} \geq S^{\prime}(0)$. We will now prove the following result.

Proposition 5.3. Let $u$ be the solution of (1.7) with a continuous and compactly supported initial condition $\phi \in \mathcal{X}_{b}(\phi \neq \equiv 0)$. Assume that $\mathcal{K}$ is given by (5.3) and that the nonlinearity $S$ satisfies Hypothesis (H2). Then, for any $\tau \geq 0$ and $\kappa \in(0,1)$, we have

$$
\frac{\rho^{*}(\tau)}{2 \alpha} \leq \liminf _{t \rightarrow \infty} \frac{\log (\min \{x: u(t, x)=\kappa\})}{t} .
$$


The combination of the lower and upper bounds shows that for sufficiently large $t$, we have

$$
\frac{\rho^{*}(\tau)}{2 \alpha} \leq \liminf _{t \rightarrow \infty} \frac{\log (\min \{x: u(t, x)=\kappa\})}{t} \leq \limsup _{t \rightarrow \infty} \frac{\log (\max \{x: u(t, x)=\kappa\})}{t} \leq \frac{\bar{\rho}(\tau)}{2 \alpha},
$$

which implies that the level sets grow exponentially fast for algebraically decaying kernels.

We will need several intermediary lemmas in order to prove the above lower bound.

Lemma 5.4. Assume that $\mathcal{K}$ is given by (5.3). For all $m \geq 1$, all $\omega>0$ and $x>0$, we have

$$
\mathcal{K}_{m}(x) \geq \frac{C_{\alpha}}{1+[x+(m-1) \omega]^{2 \alpha}}\left(\int_{-\omega}^{\omega} \mathcal{K}(y) \mathrm{d} y\right)^{m-1},
$$

where $\mathcal{K}_{m}$ is defined in (2.8).

Proof. Note first that inequality (5.7) is trivially satisfied for $m=1$. Let $m \geq 2$ and write, using Fubini's Theorem,

$$
\begin{aligned}
\mathcal{K}_{m}(x) & =\mathcal{K}_{m-1} * \mathcal{K}(x)=\int_{\mathbb{R}} \mathcal{K}_{m-1}\left(x-y_{1}\right) \mathcal{K}\left(y_{1}\right) \mathrm{d} y_{1} \\
& =\int_{\mathbb{R}}\left(\int_{\mathbb{R}} \mathcal{K}_{m-2}\left(x-y_{1}-y_{2}\right) \mathcal{K}\left(y_{2}\right) \mathrm{d} y_{2}\right) \mathcal{K}\left(y_{1}\right) \mathrm{d} y_{1} \\
& =\int_{\mathbb{R}^{m-1}} \mathcal{K}\left(x-\sum_{i=1}^{m-1} y_{i}\right) \prod_{i=1}^{m-1} \mathcal{K}\left(y_{i}\right) \mathrm{d} y_{1} \cdots \mathrm{d} y_{m-1} \\
& \geq \int_{[-\omega, \omega]^{m-1}} \mathcal{K}\left(x-\sum_{i=1}^{m-1} y_{i}\right) \prod_{i=1}^{m-1} \mathcal{K}\left(y_{i}\right) \mathrm{d} y_{1} \cdots \mathrm{d} y_{m-1} \\
& \geq \mathcal{K}\left(x+\sum_{i=1}^{m-1} \omega\right) \int_{[-\omega, \omega]} C^{m-1} \prod_{i=1}^{m-1} \mathcal{K}\left(y_{i}\right) \mathrm{d} y_{1} \cdots \mathrm{d} y_{m-1} \\
& =\frac{C_{\alpha}}{1+[x+(m-1) \omega]^{2 \alpha}}\left(\int_{-\omega}^{\omega} \mathcal{K}(y) \mathrm{d} y\right)^{m-1} \cdot
\end{aligned}
$$

Let us now introduce the following auxiliary Cauchy problem:

$$
\left\{\begin{aligned}
\partial_{t} u(t, x) & =-u(t, x)+\nu \mathcal{K} * u(t, x) & & \text { if }(t, x) \in(0, \infty) \times \mathbb{R}, \\
u(0, x) & =\varphi(x) & & \text { if } x \in \mathbb{R},
\end{aligned}\right.
$$

for any $\nu>1$, where for $\delta \in(0,1)$ we have

$$
\varphi(x)= \begin{cases}\delta, & |x| \leq 1 \\ 0, & |x|>1\end{cases}
$$

Lemma 5.5. Let $\nu>1$ and assume that $\mathcal{K}$ is given by (5.3). Then, for any $\sigma<\frac{\nu-1}{2 \alpha}$, the solution of the Cauchy problem (5.8) satisfies

$$
\lim _{t \rightarrow+\infty} \inf _{|x| \leq e^{\sigma t}} u(t, x)=+\infty .
$$


Proof. Choose $\epsilon>0$ and $\omega>0$ such that

$$
\frac{\nu-1}{2 \alpha}-\sigma>\epsilon \text { and } \int_{-\omega}^{\omega} \mathcal{K}(x) \mathrm{d} x>\nu-2 \alpha \epsilon .
$$

Note that the solution of the Cauchy problem (5.8) can be written

$$
\begin{aligned}
u(t, x) & =e^{-t} \sum_{m=0}^{\infty} \frac{\nu^{m} t^{m}}{m !} \mathcal{K}_{m} * \varphi(x) \\
& =\delta e^{-t} \sum_{m=1}^{\infty} \frac{\nu^{m} t^{m}}{m !} \int_{-1}^{1} \mathcal{K}_{m}(x-y) \mathrm{d} y+e^{-t} \varphi(x) .
\end{aligned}
$$

For $x>1$, using inequality (5.7) and the definition of $\varphi$, we obtain

$$
u(t, x) \geq \delta e^{-t} \sum_{m=1}^{\infty} \frac{\nu^{m} t^{m}}{m !}\left(\int_{-\omega}^{\omega} \mathcal{K}(y) \mathrm{d} y\right)^{m-1} \int_{-1}^{1} \frac{C_{\alpha}}{1+[(x-y)+(m-1) \omega]^{2 \alpha}} \mathrm{d} y .
$$

As a consequence,

$$
u\left(t, e^{\left(\frac{\nu-1}{2 \alpha}-\epsilon\right) t}\right) \geq \delta e^{-t} \sum_{m=1}^{\infty} \frac{\nu^{m} t^{m}}{m !}\left(\int_{-\omega}^{\omega} \mathcal{K}(y) \mathrm{d} y\right)^{m-1} \int_{-1}^{1} \frac{C_{\alpha}}{1+\left[\left(e^{\left(\frac{\nu-1}{2 \alpha}-\epsilon\right) t}-y\right)+(m-1) \omega\right]^{2 \alpha}} \mathrm{d} y
$$

and for large $t$ we have

$$
u\left(t, e^{\left(\frac{\nu-1}{2 \alpha}-\epsilon\right) t}\right) \geq \theta \sum_{m=1}^{\infty} \frac{1}{m !}\left(\nu t \int_{-\omega}^{\omega} \mathcal{K}(y) \mathrm{d} y\right)^{m} \frac{e^{(2 \alpha \epsilon-\nu) t}}{m^{2 \alpha}}
$$

for some constant $\theta>0$ which depends on $(\alpha, \delta, \omega)$. As a consequence, $u\left(t, e^{\sigma t}\right) \rightarrow+\infty$ as $t \rightarrow+\infty$ provided that

$$
\sum_{m=1}^{\infty} \frac{1}{m !}\left(\nu t \int_{-\omega}^{\omega} \mathcal{K}(y) \mathrm{d} y\right)^{m} \frac{e^{(2 \alpha \epsilon-\nu) t}}{m^{2 \alpha}} \rightarrow+\infty \text { as } t \rightarrow+\infty .
$$

It is then enough to show that for all $M>0$, there exists $T_{M}>0$ such that for all $t \geq T_{M}$, we have

$$
M e^{(\nu-2 \alpha \epsilon) t} \leq \sum_{m=1}^{\infty} \frac{1}{m !}\left(\nu t \int_{-\omega}^{\omega} \mathcal{K}(y) \mathrm{d} y\right)^{m} m^{-(2 \alpha)} .
$$

Since we have

$$
\begin{aligned}
M e^{(\nu-2 \alpha \epsilon) t} & =M \sum_{m=0}^{\infty} \frac{(\nu-2 \alpha \epsilon)^{m} t^{m}}{m !} \\
& =\frac{M}{t}\left(t+\sum_{m=1}^{\infty} \frac{(\nu-2 \alpha \epsilon)^{m} t^{m+1}}{m !}\right) \\
& t \geq T=M / \gamma \\
& \geq{ }^{\infty} \gamma\left(t+(\nu-2 \alpha \epsilon)^{-1} \sum_{m=2}^{\infty} \frac{(\nu-2 \alpha \epsilon)^{m} t^{m}}{m !} m\right)
\end{aligned}
$$

for $t \geq T=M / \gamma$, where $\gamma>0$ as to be chosen. Identifying, the first order term in (5.9), we must have

$$
\gamma \leq \nu \int_{-\omega}^{\omega} \mathcal{K}(y) \mathrm{d} y
$$


and identifying higher order terms we must also have for all $m \geq 2$

$$
(\nu-2 \alpha \epsilon)^{-1} \gamma(\nu-2 \alpha \epsilon)^{m} m \leq\left(\nu \int_{-\omega}^{\omega} \mathcal{K}(y) \mathrm{d} y\right)^{m} m^{-(2 \alpha)} .
$$

Since $\int_{-\omega}^{\omega} \mathcal{K}(x) \mathrm{d} x>\nu-2 \alpha \epsilon$ we can find $\gamma>0$ such that the above inequalities are satisfied which concludes the proof of the lemma.

Let us now introduce the delayed version of the auxiliary Cauchy problem (5.8). Namely, for $\tau>0$, let us consider

$$
\left\{\begin{aligned}
\partial_{t} u(t, x) & =-u(t, x)+\nu \mathcal{K} * u(t-\tau, x) & & \text { if }(t, x) \in(0, \infty) \times \mathbb{R} \\
u(\theta, x) & =\varphi(x) & & \text { if }(\theta, x) \in[-\tau, 0] \times \mathbb{R}
\end{aligned}\right.
$$

where for $\delta \in(0,1)$ we have

$$
\varphi(x)= \begin{cases}\delta, & |x| \leq 1 \\ 0, & |x|>1\end{cases}
$$

Lemma 5.6. Let $\nu>1$ and assume that $\mathcal{K}$ is given by (5.3). Let $\beta^{*}(\tau)>0$ be the unique solution of

$$
\beta+1=\nu e^{-\beta \tau} .
$$

Then, for any $\sigma<\frac{\beta^{*}(\tau)}{2 \alpha}$, the solution of the Cauchy problem (5.10) satisfies

$$
\lim _{t \rightarrow+\infty} \inf _{|x| \leq e^{\sigma t}} u(t, x)=+\infty
$$

Proof. The proof in the delayed case is very similar to the one without delay. The main difference being the formal representation of the solutions of the Cauchy problem (5.10), which are given through

$$
u(t, x)=\delta e^{-t} \sum_{m=1}^{\left[\frac{t}{\tau}\right]+1} \frac{e^{\tau m} \nu^{m}[t-(m-1) \tau]^{m}}{m !} \int_{-1}^{1} \mathcal{K}_{m}(x-y) \mathrm{d} y+e^{-t} \varphi(x), \quad(t, x) \in(0, \infty) \times \mathbb{R},
$$

where $\left[\frac{t}{\tau}\right]$ is the integer part of $t / \tau$. As consequence for $t$ large enough and $1 \leq|x| \leq e^{\sigma t}$ with $\sigma \in\left(0, \frac{\beta^{*}(\tau)}{2 \alpha}\right)$, we have that

$$
\begin{aligned}
u(t, x) & \geq u\left(t, e^{\sigma t}\right)=\delta e^{-t} \sum_{m=1}^{\left[\frac{t}{\tau}\right]+1} \frac{e^{\tau m} \nu^{m}[t-(m-1) \tau]^{m}}{m !} \int_{-1}^{1} \mathcal{K}_{m}\left(e^{\sigma t}-y\right) \mathrm{d} y+e^{-t} \varphi(x), \\
& \geq \tilde{\theta} e^{-(1+2 \alpha \sigma) t} \sum_{m=1}^{\left[\frac{t}{\tau}\right]+1} \frac{e^{\tau m} \nu^{m}[t-(m-1) \tau]^{m}}{m !}\left(\int_{-\omega}^{\omega} \mathcal{K}(y) \mathrm{d} y\right)^{m} m^{-2 \alpha} \\
& \geq \tilde{\theta} e^{-(1+2 \alpha \sigma) t}\left(\left[\frac{t}{\tau}\right]+1\right)^{-2 \alpha} \sum_{m=1}^{\left[\frac{t}{\tau}\right]+1} \frac{e^{\tau m} \nu^{m}[t-(m-1) \tau]^{m}}{m !}\left(\int_{-\omega}^{\omega} \mathcal{K}(y) \mathrm{d} y\right)^{m},
\end{aligned}
$$


for some constant $\tilde{\theta}>0$ which depends on $(\alpha, \delta, \omega, \tau)$. Let now denote,

$$
z_{\omega}(t):=1+\sum_{m=1}^{\left[\frac{t}{\tau}\right]+1} \frac{e^{\tau m} \nu^{m}[t-(m-1) \tau]^{m}}{m !}\left(\int_{-\omega}^{\omega} \mathcal{K}(y) \mathrm{d} y\right)^{m}, \quad t>0,
$$

then,

$$
\frac{\mathrm{d}}{\mathrm{d} t} z_{\omega}(t)=\nu\left(\int_{-\omega}^{\omega} \mathcal{K}(y) \mathrm{d} y\right) e^{\tau} z_{\omega}(t-\tau) .
$$

As a consequence, there exists $\delta_{0}>0$, such that for all $t \geq 0$,

$$
z_{\omega}(t) \geq \delta_{0} e^{\beta_{\omega}(\tau) t}
$$

where $\beta_{\omega}(\tau)$ is the unique solution of

$$
\beta+1=\nu\left(\int_{-\omega}^{\omega} \mathcal{K}(y) \mathrm{d} y\right) e^{-\beta \tau}
$$

Note that as $\omega \rightarrow+\infty$, we have $\beta_{\omega}(\tau) \rightarrow \beta^{*}(\tau)$. As a consequence, we obtain that

$$
\begin{aligned}
u(t, x) & \geq \tilde{\theta} e^{-(1+2 \alpha \sigma) t}\left(\left[\frac{t}{\tau}\right]+1\right)^{-2 \alpha}\left(z_{\omega}(t)-1\right) \\
& \geq \tilde{\theta} e^{-(1+2 \alpha \sigma) t}\left(\left[\frac{t}{\tau}\right]+1\right)^{-2 \alpha}\left(\delta_{0} e^{\beta_{\omega}(\tau) t}-1\right) .
\end{aligned}
$$

Since $\sigma \in\left(0, \frac{\beta^{*}(\tau)}{2 \alpha}\right)$, we can find $\omega$ large enough such that $\sigma<\frac{\beta_{\omega}(\tau)}{2 \alpha}$ from which one immediately obtains

$$
\lim _{t \rightarrow+\infty} \inf _{|x| \leq e^{\sigma t}} u(t, x)=+\infty
$$

The following lemma will be a key step toward the proof of Proposition 5.3 and can be thought as of an analog of Lemma 3.1 in [9].

Lemma 5.7. Assume that $\mathcal{K}$ is given by (5.3) and that the nonlinearity $S$ satisfies Hypothesis (H2) together with the condition $\frac{S(\delta)}{\delta} \leq \frac{S(u)}{u}$ for small $0 \leq u \leq \delta$. Then, for every $\sigma \in\left(0, \frac{\rho^{*}(\tau)}{2 \alpha}\right)$, there exists $t_{0} \geq 1$ and $0<\epsilon_{0}<1$ for which the following holds.

Given $r_{0} \geq 1$ and $0<\epsilon \leq \epsilon_{0}$, let $a_{0}>0$ be defined by $a_{0} r_{0}^{-2 \alpha}=\epsilon$ and let

$$
v_{0}(x)= \begin{cases}a_{0}|x|^{-2 \alpha} & \text { for }|x| \geq r_{0} \\ \epsilon & \text { for }|x| \leq r_{0}\end{cases}
$$

Then, the solution $u$ of (1.7) with initial condition $\phi(\theta, x)=v_{0}(x)$ for all $(\theta, x) \in[-\tau, 0] \times \mathbb{R}$ satisfies

$$
u\left(n t_{0}, x\right) \geq \epsilon, \quad \text { for }|x| \leq r_{0} e^{\sigma n t_{0}} \quad n \in \mathbb{N} .
$$


Proof. Let $\delta>0$ be small enough and $\omega>0$ large enough such that

$$
\sigma<\frac{\beta_{\omega}(\tau)}{2 \alpha}<\frac{\rho^{*}(\tau)}{2 \alpha}
$$

where $\beta_{\omega}(\tau)$ is the unique positive solution of

$$
\beta+1=\frac{S(\delta)}{\delta}\left(\int_{-\omega}^{\omega} \mathcal{K}(y) \mathrm{d} y\right) e^{-\beta \tau} .
$$

Define

$$
\epsilon_{0}:=\delta e^{-\left(S^{\prime}(0)-1\right) t_{0}}
$$

where $t_{0}>0$ will be specified later. For $n \geq 1$ and $\epsilon \in\left(0, \epsilon_{0}\right]$, by the semiflow property of the solution maps, we have

$$
u\left(n t_{0}, x\right)=u\left(t_{0}, x ; u\left((n-1) t_{0}, \cdot\right)\right) .
$$

As a consequence, if we define for $n \geq 1$

$$
\Psi_{n-1}:=\min \left\{\epsilon, u\left((n-1) t_{0}, \cdot\right)\right\},
$$

then we have $\Psi_{n-1} \leq \epsilon$ and by the comparison principle we obtain that

$$
u\left(t, x ; \Psi_{n-1}\right) \leq u(t, x ; \epsilon) \leq \epsilon e^{\left.\left(S^{\prime} 0\right)-1\right) t} \leq \delta
$$

for all $t \in\left[0, t_{0}\right]$. Thus, using the comparison principle once again, we have

$$
u\left(n t_{0}, x\right) \geq u\left(t_{0}, x ; \Psi_{n-1}\right) \geq v\left(t_{0}, x ; \Psi_{n-1}\right)
$$

where $v$ satisfies for all $t \in\left[0, t_{0}\right]$

$$
\partial_{t} v(t, x)=-v(t, x)+\frac{S(\delta)}{\delta} \mathcal{K} * v(t-\tau, x), \quad x \in \mathbb{R} .
$$

Using the explicit form of $v$, we obtain

$$
u\left(n t_{0}, x\right) \geq e^{-t_{0}} \sum_{m=1}^{\left[\frac{t_{0}}{\tau}\right]+1} \frac{e^{\tau m} \nu^{m}\left[t_{0}-(m-1) \tau\right]^{m}}{m !}\left(\int_{-\omega}^{\omega} \mathcal{K}(y) \mathrm{d} y\right)^{m-1} \mathcal{K} * \Psi_{n-1}(|x|+(m-1) \omega),
$$

and $\nu=\frac{S(\delta)}{\delta}$ and $\tau>0$. For $\tau=0$, we simply have

$$
u\left(n t_{0}, x\right) \geq e^{-t_{0}} \sum_{m=1}^{\infty} \frac{\nu^{m}}{m !}\left(\int_{-\omega}^{\omega} \mathcal{K}(y) \mathrm{d} y\right)^{m-1} \mathcal{K} * \Psi_{n-1}(|x|+(m-1) \omega) .
$$

From now on, we only focus on the case $\tau>0$ as the case $\tau=0$ can be handled in a similar fashion.

Claim 1: For all $|x| \geq r_{0}$, we have

$$
\mathcal{K} * \Psi_{n-1}(|x|+(m-1) \omega) \geq r_{0} \mathcal{K}\left(r_{0}+(m-1) \omega\right) \Psi_{n-1}(2|x|) .
$$


Let us postpone the proof of the claim for a moment and define

$$
A\left(t_{0}\right):=r_{0} e^{-t_{0}} \sum_{m=1}^{\left[\frac{t_{0}}{\tau}\right]+1} \frac{e^{\tau m} \nu^{m}\left[t_{0}-(m-1) \tau\right]^{m}}{m !}\left(\int_{-\omega}^{\omega} \mathcal{K}(y) \mathrm{d} y\right)^{m-1} \mathcal{K}\left(r_{0}+(m-1) \omega\right) .
$$

Then by (5.12), we have that

$$
u\left(n t_{0}, x\right) \geq A\left(t_{0}\right) \Psi_{n-1}(2|x|)
$$

for all $|x| \geq r_{0}$.

Claim 2: For all $|x| \geq r_{0}$ and $i \in \llbracket 1, n \rrbracket$, we have

$$
u\left(n t_{0}, x\right) \geq \min \left\{\epsilon A\left(t_{0}\right), \epsilon\left[A\left(t_{0}\right)\right]^{2}, \ldots, \epsilon\left[A\left(t_{0}\right)\right]^{i},\left[A\left(t_{0}\right)\right]^{i} u\left((n-i) t_{0}, 2^{i}|x|\right)\right\} .
$$

Again, let us postpone the proof. We can now find the required $t_{0}$. Indeed, using the definition of $z_{\omega}(t)$ in (5.11) from the proof of Lemma 5.6, we have that

$$
A\left(t_{0}\right) \geq r_{0}^{1-2 \alpha} e^{-t_{0}}\left(\left[\frac{t_{0}}{\tau}+1\right]\right)^{-2 \alpha}\left(z_{\omega}\left(t_{0}\right)-1\right)
$$

and thus

$$
\lim _{t_{0} \rightarrow \infty}\left(A\left(t_{0}\right) e^{-2 \alpha \sigma t_{0}}\right)=+\infty
$$

so we may assume that $A\left(t_{0}\right)>1$ for large enough $t_{0}>0$. Using this fact and equation (5.13), we see that

$$
\begin{aligned}
u\left(n t_{0}, x\right) & \geq \min \left\{\epsilon A\left(t_{0}\right),\left[A\left(t_{0}\right)\right]^{n} v_{0}\left(2^{n}|x|\right)\right\}, \quad|x| \geq r_{0} \\
& =\min \left\{\epsilon A\left(t_{0}\right), \epsilon\left[A\left(t_{0}\right)\right]^{n}\left(\frac{r_{0}}{2^{n}|x|}\right)^{2 \alpha}\right\}, \quad|x| \geq r_{0} .
\end{aligned}
$$

In particular, at $x=r_{0} e^{n \sigma t_{0}}$,

$$
\begin{aligned}
u\left(n t_{0}, r_{0} e^{n \sigma t_{0}}\right) & \geq \epsilon \min \left\{A\left(t_{0}\right),\left[A\left(t_{0}\right)\right]^{n}\left(\frac{1}{2^{n} e^{n \sigma t_{0}}}\right)^{2 \alpha}\right\} \\
& =\epsilon \min \left\{A\left(t_{0}\right),\left[A\left(t_{0}\right) \frac{1}{2^{2 \alpha}} e^{-2 \alpha \sigma t_{0}}\right]^{n}\right\} \\
& >\epsilon \min \left\{1,\left[A\left(t_{0}\right) \frac{1}{2^{2 \alpha}} e^{-2 \alpha \sigma t_{0}}\right]^{n}\right\} \\
& >\epsilon,
\end{aligned}
$$

provided that $A\left(t_{0}\right) \frac{1}{2^{2 \alpha}} e^{-2 \alpha \sigma t_{0}}>1$ which is already satisfied for large enough $t_{0}>0$, see (5.14). This concludes the proof the lemma. We now prove Claim 1 and 2. 
Proof of Claim 1: Direct computations show that for all $|x| \geq r_{0}$,

$$
\begin{aligned}
\mathcal{K} * \Psi_{n-1}(|x|+(m-1) \omega) & =\int_{\mathbb{R}} \mathcal{K}(|x|+(m-1) \omega-y) \Psi_{n-1}(y) \mathrm{d} y \\
& \geq \int_{-r_{0}+|x|}^{r_{0}+|x|} \mathcal{K}(|x|+(m-1) \omega-y) \Psi_{n-1}(y) \mathrm{d} y \\
& \geq \Psi_{n-1}(2|x|) \int_{-r_{0}+|x|}^{r_{0}+|x|} \mathcal{K}(|x|+(m-1) \omega-y) \mathrm{d} y \\
& \geq \Psi_{n-1}(2|x|) \int_{0}^{r_{0}} \mathcal{K}((m-1) \omega-y) \mathrm{d} y \\
& \geq r_{0} \mathcal{K}\left(r_{0}+(m-1) \omega\right) \Psi_{n-1}(2|x|) .
\end{aligned}
$$

Here we used the fact that $y \mapsto \Psi_{n-1}(y)$ is symmetric and decreasing on $[0, \infty)$.

Proof of Claim 2: We prove inequality (5.13) by induction. For $i=1$, we have by definition

$$
\Psi_{n-1}(2|x|)=\min \left\{\epsilon, u\left((n-1) t_{0}, 2|x|\right)\right\},
$$

and as $u\left(n t_{0}, x\right) \geq A\left(t_{0}\right) \Psi_{n-1}(2|x|)$, inequality (5.13) holds in that case. Assume that inequality (5.13) holds, that is

$$
u\left(n t_{0}, x\right) \geq \min \left\{\epsilon A\left(t_{0}\right), \epsilon\left[A\left(t_{0}\right)\right]^{2}, \ldots, \epsilon\left[A\left(t_{0}\right)\right]^{i},\left[A\left(t_{0}\right)\right]^{i} u\left((n-i) t_{0}, 2^{i}|x|\right)\right\} .
$$

Since $u\left((n-i) t_{0}, 2^{i}|x|\right) \geq A\left(t_{0}\right) \Psi_{n-i-1}\left(2^{i+1}|x|\right)=A\left(t_{0}\right) \min \left\{\epsilon, u\left((n-i-1) t_{0}, 2^{i+1}|x|\right)\right\}$, the claim is preserved.

Corollary 5.8. Assume that $\mathcal{K}$ is given by (5.3), that the nonlinearity $S$ satisfies Hypothesis (H2), and $\sigma \in\left(0, \frac{\rho^{*}(\tau)}{2 \alpha}\right)$. Let $t_{0} \geq 1$ be the time defined in Lemma 5.7. Then for every continuous and compactly supported initial condition $\phi \in \mathcal{X}_{b}(\phi \not \equiv 0)$, there exist $\epsilon \in(0,1)$ and a constant $\mathbf{b}>0$ such that

$$
u(t, x) \geq \epsilon \quad \text { for all } t \geq t_{0} \text { and }|x| \leq \mathbf{b} e^{\sigma t},
$$

where $u$ is the solution of the Cauchy problem (1.7) with initial condition $\phi$.

Proof. Let $S_{1}$ being a $\mathcal{C}^{1}$ function such that

$$
S_{1}(u) \leq S(u), \quad S_{1}^{\prime}(0)>1, \quad S_{1}^{\prime}(u) \leq S_{1}^{\prime}(0) \quad \forall u \in[0,1],
$$

so that small $0 \leq u \leq \delta$, one has $\frac{S_{1}(\delta)}{\delta} \leq \frac{S_{1}(u)}{u}$. We consider the Cauchy problem,

$$
\left\{\begin{aligned}
\partial_{t} \underline{\mathrm{u}}_{1}(t, x) & =-\underline{\mathrm{u}}_{1}(t, x)+\int_{\mathbb{R}} \mathcal{K}(x-y) S_{1}\left(\underline{\mathrm{u}}_{1}(t-\tau, y)\right) \mathrm{d} y & & \text { if }(t, x) \in(0, \infty) \times \mathbb{R}, \\
\underline{\mathrm{u}}_{1}(t, x) & =\phi(t, x) & & \text { if }(t, x) \in[-\tau, 0] \times \mathbb{R} .
\end{aligned}\right.
$$

Using, the comparison principle for delayed equations (see e.g., [35]) we have that

$$
u(t, x) \geq \underline{\mathrm{u}}_{1}(t, x) \quad \text { for all }(t, x) \in(0, \infty) \times \mathbb{R} .
$$


Furthermore, using the fact that $f_{1}(u):=-u+S_{1}(u) \geq 0$ for all $u \in[0,1]$, we have

$$
\partial_{t} \underline{\mathrm{u}}_{1}(t, x)=-\underline{\mathrm{u}}_{1}(t, x)+\int_{\mathbb{R}} \mathcal{K}(x-y) S_{1}\left(\underline{\mathrm{u}}_{1}(t-\tau, y)\right) \mathrm{d} y \geq-\underline{\mathrm{u}}_{1}(t, x)+\int_{\mathbb{R}} \mathcal{K}(x-y) \underline{\mathrm{u}}_{1}(t-\tau, y) \mathrm{d} y .
$$

We denote by $Q_{t}[\phi]$ the solution semiflow associated to the following Cauchy problem

$$
\left\{\begin{aligned}
\partial_{t} u(t, x) & =-u(t, x)+\int_{\mathbb{R}} \mathcal{K}(x-y) u(t-\tau, y) \mathrm{d} y & & \text { if }(t, x) \in(0, \infty) \times \mathbb{R}, \\
u(t, x) & =\phi(t, x) & & \text { if }(t, x) \in[-\tau, 0] \times \mathbb{R} .
\end{aligned}\right.
$$

Using once more the comparison principle we have that

$$
\underline{\mathrm{u}}_{1}(t, x) \geq Q_{t}[\phi](x) \quad \text { for all }(t, x) \in(0, \infty) \times \mathbb{R} .
$$

And thus, as $\phi \not \equiv 0$, we have that $\underline{\underline{u}}_{1}\left(t_{0} / 2, \cdot\right) \geq Q_{t_{0} / 2}[\phi]>0$ in $\mathbb{R}$. Since $Q_{t_{0} / 2}[\phi]$ is a positive continuous function, there exists $\delta>0$ such that $Q_{t_{0} / 2}[\phi] \geq \delta \mathbb{1}_{[-1,1]}$ in $\mathbb{R}$. Therefore,

$$
\underline{\mathrm{u}}_{1}\left(t_{0} / 2+t, x\right) \geq Q_{t}\left[\delta \mathbb{1}_{[-1,1]}\right](x), \quad(t, x) \in(0, \infty) \times \mathbb{R} .
$$

We now use the representation formula that we already obtained in Lemma 5.6 with $\nu=1$

$$
Q_{t}\left[\delta \mathbb{1}_{[-1,1]}\right](x)=\delta e^{-t} \sum_{m=1}^{\left[\frac{t}{\tau}\right]+1} \frac{e^{\tau m}[t-(m-1) \tau]^{m}}{m !} \int_{-1}^{1} \mathcal{K}_{m}(x-y) \mathrm{d} y+\delta e^{-t} \mathbb{1}_{[-1,1]}(x),
$$

for all $(t, x) \in(0, \infty) \times \mathbb{R}$. By Lemma 5.4 and the estimate for $z_{\omega}(t)$ defined in (5.11), we can find some $r_{0} \geq 1$ which depends on $t_{0}$ such that

$$
Q_{t}\left[\delta \mathbb{1}_{[-1,1]}\right](x) \geq a_{0}|x|^{-2 \alpha}, \quad \text { for } t \in\left[t_{0} / 2,3 t_{0} / 2\right] \quad \text { and }|x| \geq r_{0},
$$

for some constant $a_{0}>0$, where $t_{0} \geq 1$ is the time defined in Lemma 5.7. We can take $a_{0}$ even smaller to make $\epsilon:=a_{0} r_{0}^{-2 \alpha} \leq \epsilon_{0}$ where $\epsilon_{0}>0$ is given in Lemma 5.7. As a consequence, we deduce that

$$
u\left(t_{0} / 2+t, \cdot\right) \geq Q_{t}\left[\delta \mathbb{1}_{[-1,1]}\right] \geq v_{0} \quad \text { in } \mathbb{R},
$$

for all $t \in\left[t_{0} / 2,3 t_{0} / 2\right]$ and $v_{0}$ is the initial condition given in Lemma 5.7.

Thus, we can apply Lemma 5.7 to get a lower bound for $u\left(\cdot+T_{0}, \cdot\right)$ for all $T_{0} \in\left[t_{0}, 2 t_{0}\right]$. Since $\left\{T_{0}+n t_{0} \mid n \in \mathbb{N}\right.$ and $\left.T_{0} \in\left[t_{0}, 2 t_{0}\right]\right\}$ covers all $\left[t_{0},+\infty\right)$, we have

$$
u(t, x) \geq \epsilon \quad \text { if } t \geq t_{0} \quad \text { and }|x| \leq r_{0} e^{-\sigma 2 t_{0}} e^{\sigma t} .
$$

This finishes the proof of the corollary by taking $\mathbf{b}:=r_{0} e^{-\sigma 2 t_{0}}$.

Lemma 5.9. Assume that $\mathcal{K}$ is given by (5.3), that the nonlinearity $S$ satisfies Hypothesis (H2). Let $u$ be a solution of the Cauchy problem (1.7) with initial condition $\phi \in \mathcal{X}_{b}(\phi \neq \equiv)$ and

$$
u(t, x) \geq \epsilon \quad \text { for all } t \geq t_{0} \text { and }|x| \leq \widetilde{\mathbf{b}} e^{\sigma t},
$$

for some positive constants $\epsilon \in(0,1), \widetilde{\mathbf{b}}, \sigma$ and $t_{0}$. Then, we have for all $\kappa \in(0,1)$, there exist constants $T_{\kappa}>t_{0}$ and $C_{\kappa}>0$ such that

$$
u(t, x) \geq \kappa \quad \text { for all } t \geq T_{\kappa} \text { and }|x| \leq \frac{1}{C_{\kappa}} e^{\sigma t} .
$$


Proof. Let fix $\kappa \in(0,1)$ and choose $M>0$ large enough such that

$$
\sup _{u \in(0,1)}\left\{\left(\int_{-M}^{M} \mathcal{K}(y) \mathrm{d} y\right) S(u)>u\right\}=\frac{\kappa+1}{2} .
$$

Let $\underline{u}_{M}$ be the solution of the Cauchy problem

$$
\left\{\begin{aligned}
\partial_{t} \underline{u}_{M}(t, x) & =-\underline{u}_{M}(t, x)+\int_{\mathbb{R}} \mathcal{K}^{M}(x-y) S\left(\underline{u}_{M}(t-\tau, y)\right) \mathrm{d} y & & \text { if }(t, x) \in(0, \infty) \times \mathbb{R}, \\
\underline{u}_{M}(t, x) & =\psi_{M}(x) & & \text { if }(t, x) \in[-\tau, 0] \times \mathbb{R},
\end{aligned}\right.
$$

where $\mathcal{K}^{M}=\mathcal{K} \mathbb{1}_{[-M, M]}$ and $\psi_{M}(x)=0$ for all $|x| \geq M$ and $0<\psi_{M}(x) \leq \epsilon$ for all $|x|<M$. Then $\underline{u}_{M}$ is a sub-solution of (1.7). Moreover, by assumption we have

$$
u(t, x) \geq \epsilon \quad \text { for all } t \geq t_{0} \text { and }|x| \leq \widetilde{\mathbf{b}} e^{\sigma t}
$$

which implies that

$$
u(t, x) \geq \psi_{M}(x+y) \quad \text { for all } x \in \mathbb{R}, \quad t \geq t_{0} \text { and }|y| \leq \widetilde{\mathbf{b}} e^{\sigma t}-1 .
$$

Furthermore, note that when $|x| \geq \widetilde{\mathbf{b}} e^{\sigma t}$, we have

$$
|x+y| \geq|x|-|y| \geq \widetilde{\mathbf{b}} e^{\sigma t}-|y| \geq 1,
$$

which then implies that $\psi_{M}(x+y)=0$ in that region. Therefore, by the comparison principle and the semi-group property, we have

$$
u(t+s, x) \geq \underline{u}_{M}(s, x+y), \quad \text { for all } x \in \mathbb{R}, \quad t \geq t_{0}+\tau, \quad s \geq 0, \quad \text { and }|y| \leq \widetilde{\mathbf{b}} e^{\sigma t}-1 .
$$

Since $\underline{u}_{M}$ has a spreading property, we obtain in particular that

$$
\lim _{t \rightarrow+\infty|x|<c t} \inf _{M}(t, x)=\frac{\kappa+1}{2}, \quad c<c_{M}^{*} .
$$

And as a consequence, there exists $s_{0}>0$ such that

$$
\underline{u}_{M}\left(s_{0}, 0\right) \geq \kappa, \quad s \geq s_{0},
$$

and thus

$$
u(t+s,-y) \geq \underline{u}_{M}\left(s_{0}, 0\right) \geq \kappa, \quad \text { for all } t \geq t_{0}+\tau, \quad s \geq s_{0}, \quad \text { and }|y| \leq \widetilde{\mathbf{b}} e^{\sigma t}-1 .
$$

In particular,

$$
u\left(t+s_{0},-y\right) \geq \kappa, \quad \text { for all } t \geq t_{0}+\tau, \text { and }|y| \leq \widetilde{\mathbf{b}} e^{\sigma t}-1 .
$$

By change of variable, we obtain

$$
u(t, y) \geq \kappa \quad \text { for all } t \geq t_{0}+s_{0}+\tau \text { and }|y| \leq \widetilde{\mathbf{b}} e^{\sigma t}-1 .
$$

The proof is thus complete. 
Proof. [Proof of Proposition 5.3] The combination Corollary 5.8 and 5.9 shows that for any $\tau \geq 0$ and any $\kappa \in(0,1)$ there is $T_{\kappa}^{\tau}>0$ such that for all $t \geq T_{\kappa}^{\tau}$

$$
u(t, x) \geq \kappa \quad \text { for all } t \geq T_{\kappa} \text { and }|x| \leq \frac{1}{C_{\kappa}} e^{\sigma t} .
$$

And thus, we have

$$
\frac{\rho^{*}(\tau)}{2 \alpha} \leq \liminf _{t \rightarrow \infty} \frac{\log (\min \{x: u(t, x)=\kappa\})}{t} .
$$

This completes the proof of Proposition 5.3.

\section{Discussion}

Summary of results. In this paper, we have studied the existence of traveling wave solutions and spreading properties for single-layer delayed neural field equations when the kinetic dynamics are of monostable type. We have characterized the invasion speeds as a function of the time delay when the connectivity kernel is exponentially bounded and for nonlinearities of KPP type. As expected, we recovered the fact that delay slows down the wave speed. Our results rely on the abstract monotone dynamical systems approach developed in [17, 27, 28]. We have also shown that for exponentially unbounded kernels the level sets of the solutions propagate with an infinite asymptotic speed. In the specific case of algebraically decaying kernels, we were able to find lower and upper bounds for the position of any level sets and proved that this position moves exponentially fast as time goes to infinity.

Towards heterogeneous neural fields. This work is a first step toward a more systematic study of generalization of equations (1.1) including space-time heterogeneities of the form

$$
\partial_{t} u(t, x)=-u(t, x)+\int_{\mathbb{R}} \mathcal{K}(x-y, y, t) S(u(t-\tau, y)) \mathrm{d} y, \quad(t, x) \in(0, \infty) \times \mathbb{R},
$$

where, for example, the connectivity function $\mathcal{K}$ could be $L$-periodic in its second argument and $T$-periodic in its third. Such equations were partially analyzed in $[7,13]$ in the context of spaceperiodic heterogeneities motivated by the modeling of the periodic micro-structure of the visual cortex. The recent theoretical developments introduced in [19] should provide some interesting directions for the analysis of spreading properties and traveling wave solutions of equations of the form (6.1).

Towards more realistic transmission delays. From a modeling point of view, the form of the delays that we considered in our neural field equation (1.1) only takes into account synaptic delays [33]. However, it is well known that transmission delays, due to the propagation of action potentials along axons, are also crucial from a modeling point of view [39-41] and play an important role on 
the spatiotemporal dynamics of cortical areas. Neural field equations with transmission delays are often written in the following form

$$
\partial_{t} u(t, x)=-u(t, x)+\int_{\mathbb{R}} \mathcal{K}(x-y) S(u(t-d(x-y), y)) \mathrm{d} y, \quad(t, x) \in(0, \infty) \times \mathbb{R},
$$

where the function $z \rightarrow d(z)$ is defined as

$$
d(z):= \begin{cases}\tau+\frac{|z|}{c}, & |z| \geq \ell \\ \tau+\frac{\ell}{c}, & |z|>\ell\end{cases}
$$

$\tau>0$ is the synaptic delay, $\ell>0$ represents the maximum spatial extent where delays are taken into account and $c>0$ is the transmission wave speed that is of the order of 0.1 to $0.3 \mathrm{~ms}^{-1}$ [8]. To the best of our knowledge, most of studies have focused so far on the stability and bifurcation analysis of stationary solutions of (6.2) [39-41]. We believe that the theoretical treatment of spreading properties and traveling wave solutions of such equations is an open challenge and will be the subject of our future investigations.

\section{Acknowledgments}

We express our gratitude to the anonymous referee for a careful reading and helpful suggestions which led to an improvement of our original manuscript. The research leading to these results has received funding from the European Research Council under the European Union's Seventh Framework Program (FP/2007-2013) / ERC Grant Agreement n321186 : "Reaction-Diffusion Equations, Propagation and Modelling" led by H. Berestycki. JF is also supported in part by the NSF of China (No.11371111) and the Fundamental Research Funds for the Central Universities of China (AUGA5710050313).

\section{References}

[1] M. Aguerrea, C. Gomez, S. Trofimchuk. On uniqueness of semi-wavefronts, Diekmann-Kaper theory of a nonlinear convolution equation re-visited. Mathematische Annalen, vol 354, Issue 1, pp. 73-109, 2012.

[2] S.-I. Amari. Dynamics of pattern formation in lateral-inhibition type neural fields. Biological Cybernetics, 27(2):77-87, 1977.

[3] D.G. Aronson and H.F. Weinberger.Multidimensional nonlinear diffusion arising in population genetics, Adv. Math. 30 (1976), pp. 33-76.

[4] A. Benucci, R. A. Frazor, and M. Carandini. Standing waves and traveling waves distinguish two circuits in visual cortex. Neuron, 55, pages 103-17, 2007. 
[5] P.C. Bressloff. Waves in Neural Media: From single Neurons to Neural Fields. Lecture notes on mathematical modeling in the life sciences, Springer, 2014.

[6] P.C. Bressloff. Spatiotemporal Dynamics of Continuum Neural Fields. J. Phys. A: Math. Theor., vol 45, 109pp, 2012.

[7] P.C. Bressoll, From invasion to extinction in heterogeneous neural fields, J. of Math. Neur., (2012) 2:6.

[8] J.M.L. Budd, K. Kovács, A.S. Ferecskó, P. Buzás, U.T. Eysel, et al. Neocortical axon arbors trade-off material and conduction delay conservation. PLoS Comput. Biol. 6 (3), 2010.

[9] X. Cabré and J-M. Roquejoffre. The influence of fractional diffusion in Fisher-KPP equations. Commun. Math. Phys. 320, pp. 679-722, 2013.

[10] J. Carr and A. Chmaj. Uniqueness of traveling waves for nonlocal monostable equations. Proc. Amer. Math. Soc., vol 132, no 8, pp. 2433-2439, 2004.

[11] J.S. Clark. Why trees migrate so fast: Confronting theory with dispersal biology and the paleorecord. American Naturalist, 152, pp. 204-224, 1998.

[12] S. Coombes. Waves, bumps, and patterns in neural fields theories. Biological Cybernetics, vol. 93, no. 2, pages 91-108, 2005.

[13] S. Coombes and C.R. Laing, Pulsating fronts in periodically modulated neural field models, Phys Rev E, (2011), 83:011912.

[14] O. Diekmann and H.G. Kaper. On the bounded solutions of a nonlinear convolution equation. Nonlinear Analysis: TMA, vol 2, num 6, pp. 721-737, 1978.

[15] G. B. Ermentrout and J. B. McLeod. Existence and uniqueness of travelling waves for a neural network. Proc. Roy. Soc. Edin., 123A, pp. 461-478, 1993.

[16] J. Fang and X.-Q. Zhao. Existence and uniqueness of traveling waves for non-monotone integral equations with applications. J. Differential Equations 248 (2010), no. 9, 2199-2226.

[17] J. Fang and X.-Q. Zhao. Traveling waves for monostable semiflows with weak compactness. SIAM J. Math. Anal. vol 46, No 6, pp. 3678-3704, 2014.

[18] J. Fang, K. Lan, G. Seo and J. Wu. Spatial dynamics of an age-structured population model of asian clams. SIAM J. Appl. Math., vol 74, No 4, pp. 959-979, 2014.

[19] J. Fang, X. Yu, X.-Q. Zhao. Traveling waves and spreading speeds for time-space periodic monotone systems. submitted, 2015.

[20] G. Faye and A. Scheel, Existence of pulses in excitable media with nonlocal coupling, Adv. Math., vol 230, pp. 400-456, 2015. 
[21] G. Faye, Existence and stability of traveling pulses of a neural field equation with synaptic depression, SIAM J. Appl. Dyn. Syst, 12-4, pp. 2032-2067, 2013.

[22] R. A. Fisher, The wave of advance of advantageous genes, Ann. of Eugenics 7 , pp. 355-369, 1937.

[23] J. Garnier, Accelerating solutions in integro-differential equations, SIAM J. Math. Anal., vol 43, no 4, pp. 1955-1974, 2011.

[24] X. Huang, W. C. Troy, Q. Yang, H. Ma, C. R. Laing, S. J. Schiff, and J.-Y. Wu. Spiral waves in disinhibited mammalian neocortex. J. Neurosci., 24, pages 9897-9902, 2007.

[25] X. Huang, W. Xu, J. Liang, K. Takagaki, X. Gao and J.-Y. Wu. Spiral wave dynamics in neocortex. Neuron, 69, pages 978-990, 2010.

[26] A. N. Kolmogorov, I. G. Petrovsky and N. S. Piskunov, Etude de l'équation de la diffusion avec croissance de la quantité de matière et son application à un problème biologique, Bulletin Université d'Etat Moscou, Bjul. Moskowskogo Gos. Univ., pp. 1-26, 1937.

[27] X. Liang and X.-Q. Zhao. Asymptotic speeds of spread and traveling waves for monotone semiflows with applications. Comm. Pure appl. Math., 60, pp. 1-40, 2007.

[28] X. Liang and X.-Q. Zhao. Spreading speeds and traveling waves for abstract monostable evolution systems. J. Funct. Anal., 259, pp. 857-903, 2010.

[29] D.J. Pinto, S. L. Patrick, W. C. Huang, and B. W. Connors. Initiation, propagation, and termination of epileptiform activity in rodent neocortex in vitro involve distinct mechanisms. Journal of Neuroscience, 25, pages 8131-8140, 2005.

[30] S. Ma and J. Wu. Existence, uniqueness and asymptotic stability of traveling wavefronts in a non-local delayed diffusion equation. Journal of Dynamics and Differential Equations, 19(2), pp. 391-436, 2007.

[31] R. H. Martin and H. L. Smith, Abstract functional differential equations and reaction-diffusion systems. Tran. Amer. Math. Soc., 321, pp. 1-44, 1990.

[32] N. Rawal and W. Shen, Criteria for the existence and lower bounds of principle eigenvalues of time periodic nonlocal dispersal operators and applications. J. Dyn. Diff. Eqns, 24, 927-954, 2012 .

[33] A. Roxin and E. Montbrio. How effective delays shape oscillatory dynamics in neuronal networks. Physica D: Nonlinear Phenomena, 240(3):323-345, 2011.

[34] T.K. Sato, I. Nauhaus and M. Carandini. Traveling waves in visual cortex. Neuron Review, 75, pages 218-229, 2012. 
[35] H.L. Smith. Monotone dynamical systems: An introduction to the theory of competitive and cooperative systems. AMS, Providence, RI, 1995.

[36] J. W.-H. So, J. Wu and X. Zou. A reaction-diffusion model for a single species with age structure. I Travelling wavefronts on unbounded domains. Proc. R. Soc. Lond. A, 457, pp. 1841-1853, 2001.

[37] H.R. Thieme. Asymptotic estimates of the solutions of nonlinear integral equations and asymptotic speeds for the spread of populations. J. reine angew. Math, 306(94), 21, 1979.

[38] H. R. Thieme and X.Q. Zhao. Asymptotic speeds of spread and traveling waves for integral equations and delayed reaction-diffusion models. Journal of Differential Equations, 195(2), pp. 430-470, 2003.

[39] S. A van Gils, S. G Janssens, Y. A Kuznetsov, and S. Visser. On local bifurcations in neural field models with transmission delays. Journal of mathematical biology, pp. 1-51, 2013.

[40] R. Veltz and O. Faugeras. Stability of the stationary solutions of neural field equations with propagation delays. The Journal of Mathematical Neuroscience, 1(1):1 2011.

[41] R. Veltz and O. Faugeras. A center manifold result for delayed neural fields equations. SIAM Math. Analysis, 45(3):1527-1562, 2013.

[42] R. Veltz. An analytical method for computing Hopf bifurcation curves in neural field networks with space-dependent delays. C. R. Acad. Sci. Paris, Ser. I 349, pp. 749-752, 2011.

[43] H. Weinberger. Long-time behavior of a class of biological models. SIAM J. Math. Anal., 13 (1982), pp. 353-396.

[44] H. Weinberger and X.Q. Zhao. An extension of the formula for spreading speeds. Math. Biosci. Eng., 7, pp. 187-194, 2010.

[45] P. Weng and X.-Q. Zhao. Spreading speed and traveling waves for a multi-type SIS epidemic model. J. Differential Equations 229, no. 1, pp. 270-296, 2006.

[46] H.R. Wilson and J.D. Cowan. A mathematical theory of the functional dynamics of cortical and thalamic nervous tissue. Biological Cybernetics, 13(2):55-80, 1973.

[47] J.-Y. Wu, X. Huang and C. Zhang. Propagating waves of activity in the neocortex: What they are, what they do. Neuroscientist, 14, pages 487-502, 2008.

[48] W. Xu, X. Huang, K. Takagaki, and J.-Y. Wu. Compression and reflection of visually evoked cortical waves. Neuron, 55, pages 119-129, 2007.

[49] T. Yi and X. Zou. Global dynamics of a delay differential equation with spatial non-locality in an unbounded domain. J. Differential Equations, 251, pages 2598-2611, 2011. 